\title{
CALCULATION OF THE CONSTANT FACTOR IN THE SIX-VERTEX MODEL
}

\author{
PAVEL BLEHER AND THOMAS BOTHNER
}

\begin{abstract}
We calculate explicitly the constant factor $C$ in the large $N$ asymptotics of the partition function $Z_{N}$ of the six-vertex model with domain wall boundary conditions on the critical line between the disordered and ferroelectric phases. On the critical line the weights $a, b, c$ of the model are parameterized by a parameter $\alpha>1$, as $a=\frac{\alpha-1}{2}, b=\frac{\alpha+1}{2}, c=1$. The asymptotics of $Z_{N}$ on the critical line was obtained earlier in the paper [8] of Bleher and Liechty: $Z_{N}=$ $C F^{N^{2}} G^{\sqrt{N}} N^{1 / 4}\left(1+O\left(N^{-1 / 2}\right)\right)$, where $F$ and $G$ are given by explicit expressions, but the constant factor $C>0$ was not known. To calculate the constant $C$, we find, by using the Riemann-Hilbert approach, an asymptotic behavior of $Z_{N}$ in the double scaling limit, as $N$ and $\alpha$ tend simultaneously to $\infty$ in such a way that $\frac{N}{\alpha} \rightarrow t \geq 0$. Then we apply the Toda equation for the tau-function to find a structural form for $\stackrel{\alpha}{C}$, as a function of $\alpha$, and we combine the structural form of $C$ and the double scaling asymptotic behavior of $Z_{N}$ to calculate $C$.
\end{abstract}

\section{InTRODUCTION AND SUMMARY OF RESUlTS}

The six-vertex model is a model in statistical mechanics stated on a square lattice in $\mathbb{Z}^{2}$ with $N^{2}$ vertices and arrows on edges. The arrows obey the ice-rule: at every vertex two arrows point in and two arrows point out. This rule allows for six possible configurations which are depicted in Figure 1.

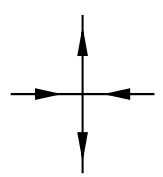

(1)

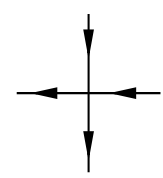

(4)

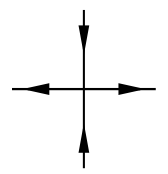

(2)

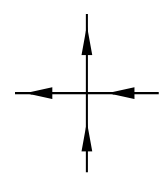

(5)

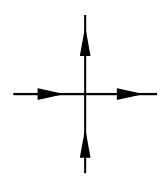

(3)

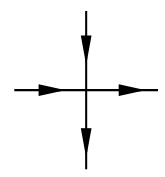

(6)

Figure 1. The arrow configurations at a vertex allowed by the ice-rule

Date: October 4, 2018.

2010 Mathematics Subject Classification. Primary 82B23; Secondary 15B52.

Key words and phrases. Six-vertex model, domain wall boundary conditions, critical line between disordered and antiferroelectric phases, asymptotic behavior of the partition function, Riemann-Hilbert problem, Deift-Zhou nonlinear steepest descent method, Toda equation.

The first author is supported in part by the National Science Foundation (NSF) Grants DMS-0969254 and DMS1265172 . 
On the lattice boundary we consider domain wall boundary conditions (DWBC), in which all arrows on the top and bottom side of the lattice point inside, and all arrows on the left and right side point outside. We depict a possible $4 \times 4$ configuration with DWBC in Figure 2 below.

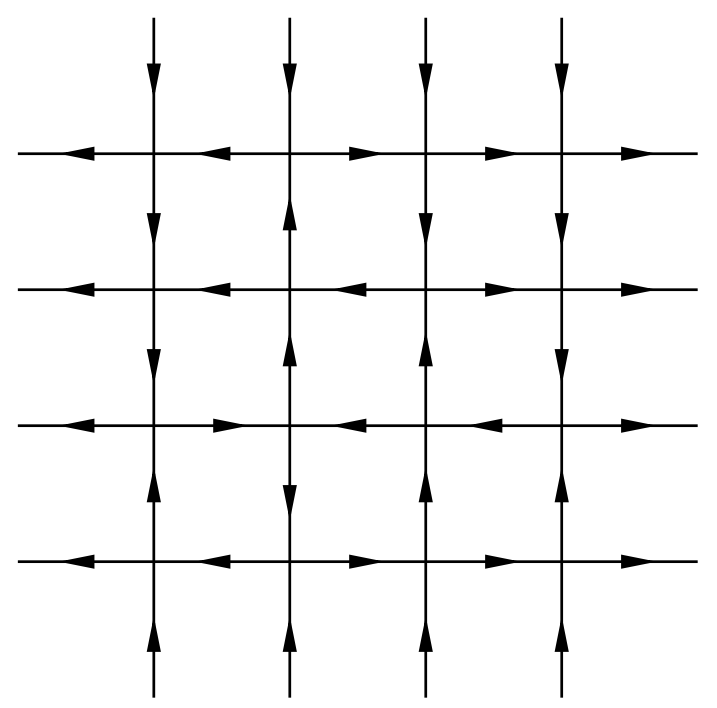

Figure 2. One example of a $4 \times 4$ configuration with DWBC

For each vertex type $(i)$ shown in Figure 1, we assign a Boltzmann weight $w_{i}, i=1, \ldots 6$ and introduce the partition function $Z_{N}$ as

$$
Z_{N}=\sum_{\text {configurations }} \prod_{i=1}^{6} w_{i}^{n_{i}},
$$

where $n_{i}$ denotes the number of vertices of type $(i)$. The partition function $Z_{N}$ depends, by definition, on six parameters: the weights $w_{i}$. Through the existence of conservation laws (compare [1, 6] or [20]) we can reduce the general case to the case when $w_{1}=w_{2}=a, w_{3}=w=4=b$, and $w_{5}=w_{6}=c$. By using the homogeneity of the partition function with respect to $a, b$, and $c$, it can be further reduced to two parameters, $\frac{a}{c}$ and $\frac{b}{c}$.

The phase diagram of the model is depicted in Figure 3, it shows three phase regions: the antiferroelectric (AF) phase region, the disordered (D) phase region, and the ferroelectric (F) phase region. In these phase regions the weights $a, b$ and $c$ are parameterized as follows:

$$
\begin{array}{llll}
a=\sinh (t-\gamma), & b=\sinh (t+\gamma), & c=\sinh (2 \gamma), & 0<|\gamma|<t \\
a=\sinh (\gamma-t), & b=\sinh (\gamma+t), & c=\sinh (2 \gamma), & 0 \leq|t|<\gamma \\
a=\sin (\gamma-t), & b=\sin (\gamma+t), & c=\sin (2 \gamma), & 0 \leq|t|<\gamma<\frac{\pi}{2} .
\end{array}
$$

In the present work we calculate the asymptotics of the partition function $Z_{N}$ of the six-vertex model on the critical line between the disordered and ferroelectric phases which corresponds to

$$
\frac{b}{c}-\frac{a}{c}=1 \text {. }
$$

On the critical line we use the following parameterization of the weights:

$$
a=\frac{\alpha-1}{2}, \quad b=\frac{\alpha+1}{2}, \quad c=1, \quad \alpha \in(1, \infty) .
$$




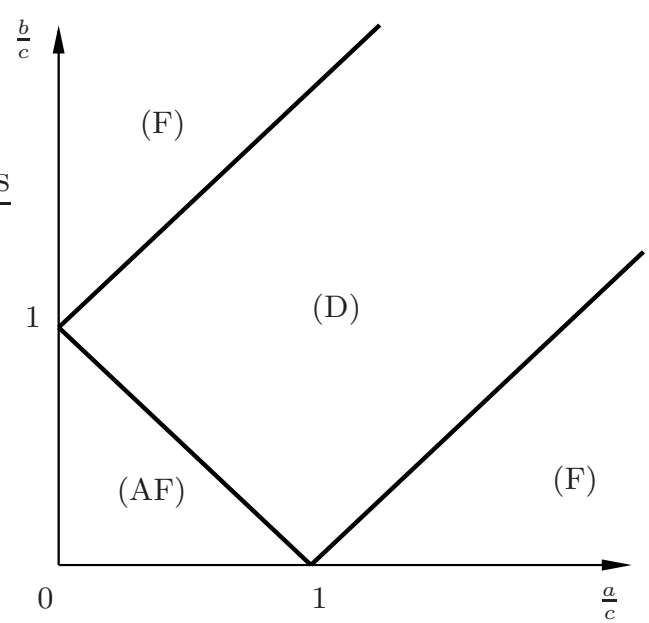

Figure 3. The phase diagram of the six-vertex model, with the phases (F), (AF) and (D)

The six-vertex model with DWBC was first studied by Korepin in 24, then further analyzed in the works [23] and [13]. This effort lead to a determinantal formula for the partition function $Z_{N}$, which is commonly called the Izergin-Korepin formula. On the D-F critical line, with weights (1.5), the Izergin-Korepin formula is

$$
Z_{N}(a, b, c)=\left(\frac{\alpha^{2}-1}{2}\right)^{N^{2}} \frac{\tau_{N}}{\left(\prod_{k=0}^{N-1} k !\right)^{2}},
$$

where $\tau_{N}$ is the Hankel determinant,

$$
\tau_{N}=\operatorname{det}\left(\frac{d^{i+j-2}}{d \alpha^{i+j-2}} \varphi\right)_{i, j=1}^{N}, \quad \varphi(\alpha)=\frac{2}{\alpha^{2}-1} .
$$

Also, as a consequence of the determinantal formula, $\tau_{N}$ satisfies the Toda equation,

$$
\tau_{N} \tau_{N}^{\prime \prime}-\left(\tau_{N}^{\prime}\right)^{2}=\tau_{N+1} \tau_{N-1}, \quad N \geq 1, \quad\left(^{\prime}\right)=\frac{d}{d \alpha} .
$$

It was observed by Zinn-Justin [28] that the Hankel determinant $\tau_{N}$ can be connected to orthogonal polynomials: Since

$$
\varphi(\alpha)=\frac{1}{\alpha-1}-\frac{1}{\alpha+1}=\int_{0}^{\infty} e^{-\alpha x}\left(e^{x}-e^{-x}\right) d x
$$

we deduce the Zinn-Justin formula,

$$
\tau_{N}=\frac{1}{N !} \int_{0}^{\infty} \ldots \int_{0}^{\infty} \prod_{j=1}^{N} w\left(x_{j}\right) \prod_{i<j}\left(x_{i}-x_{j}\right)^{2} d x_{1} \cdots d x_{N},
$$

where

$$
w(x)=e^{-\alpha x}\left(e^{x}-e^{-x}\right)
$$


Now introduce monic orthogonal polynomials $\left\{p_{n}(x)=x^{n}+\ldots\right\}_{n \geq 0}$ with respect to the measure $d \mu(x)=w(x) d x$ on the half-axis $[0, \infty)$,

$$
\int_{0}^{\infty} p_{n}(x) p_{m}(x) d \mu(x)=h_{n} \delta_{n m}
$$

and obtain from (1.9) via orthogonality, that

$$
\tau_{N}=\prod_{k=0}^{N-1} h_{k}
$$

The latter identity allows us to rewrite the Toda equation (1.7) on the critical line in the convenient form

$$
\left(\ln \tau_{N}\right)^{\prime \prime}=\frac{h_{N}}{h_{N-1}}, \quad\left({ }^{\prime}\right)=\frac{d}{d \alpha},
$$

and for the partition function, via (1.6),

$$
Z_{N}=\left(\frac{\alpha^{2}-1}{2}\right)^{N^{2}} \prod_{k=0}^{N-1} \frac{h_{k}}{(k !)^{2}} .
$$

In [8], Bleher and Liechty derive the following large $N$ asymptotics of the partition function $Z_{N}$ :

Theorem 1.1 (see [8]). On the critical line between the disordered and ferroelectric phase region with $\alpha>1$, as $N \rightarrow \infty$,

$$
Z_{N}=C F^{N^{2}} G^{\sqrt{N}} N^{1 / 4}\left(1+O\left(N^{-1 / 2}\right)\right),
$$

with

$$
F=\frac{\alpha+1}{2}, \quad G=\exp \left[-\zeta\left(\frac{3}{2}\right) \sqrt{\frac{\alpha-1}{2 \pi}}\right] .
$$

Here $\zeta(s)$ denotes the Riemann zeta function, $C>0$, and the error term in (1.15) is uniform on any compact subset of the set

$$
\{\alpha \in \mathbb{R}: \alpha>1\} .
$$

The main result in the present paper is an explicit evaluation of the constant factor $C>0$. We prove the following result:

Theorem 1.2. The constant factor $C$ in asymptotic formula (1.15) is equal to

$$
C=e^{c}(\alpha-1)^{1 / 4},
$$

where

$$
c=\frac{1}{4} \ln 2+\frac{1}{2} \ln \pi+\frac{1}{4 \pi} \sum_{n=1}^{\infty}\left[-\frac{\pi}{n}+\sum_{m=1}^{\infty} \frac{1}{(m+n) \sqrt{m n}}\right] .
$$

Remark: Using MAPLE for the numerical evaluation of the series, we obtain that

$$
\sum_{n=1}^{\infty}\left[-\frac{\pi}{n}+\sum_{m=1}^{\infty} \frac{1}{(m+n) \sqrt{m n}}\right]=-3.568781612 \ldots
$$

and

$$
c=0.4616571210 \ldots
$$


The calculation of the constant factor in the asymptotics of the partition function is a notoriously difficult problem. This problem appears not only in exactly solvable models of statistical mechanics, such as the six-vertex model and the Ising model, but also in random matrix theory, combinatorics, theory of integrable PDEs, etc. In different settings, the "constant factor problem" is studied in the works of Tracy [26, Basor and Tracy [3], Budylin and Buslaev [5], Ehrhardt [19], Deift, Its, Krasovsky, and Zhou [14], Deift, Its, and Krasovsky [15], Baik, Buckingham, and DiFranco [2], Bothner and Its [11, Forrester [22], and others.

To prove Theorem 1.2, we develop the Riemann-Hilbert approach to the double scaling limit of the partition function $Z_{N}$, as both $N$ and $\alpha$ tend to $\infty$ in such a way that $\frac{N}{\alpha} \rightarrow t \geq 0$, see Theorem 6.1 below. The double scaling asymptotics of the partition function can be of interest by itself. Then we use the Toda equation to show that the constant $C$ can be written as

$$
C=(\alpha-1)^{1 / 4} e^{d \alpha+c} .
$$

After that we apply the double scaling asymptotics of $Z_{N}$ to calculate the values of $d$ and $c$. In this way we find that $d=0$ and that $c$ is given by formula (1.18).

The result of Theorem [1.2 adds to the work of the first author with Vladimir Fokin [6], with Karl Liechty [7, 8, 9], and with the second author [5]. This series of papers proves conjectures of Paul Zinn-Justin 28] on the large $N$ asymptotics of $Z_{N}$ in the phase regions. For the convenience of the reader, we briefly summarize obtained results and outline what is known about the constant factor in different phase regions.

Ferroelectric phase region. With parameterization (1.1), for any $\varepsilon>0$,

$$
Z_{N}=C F^{N^{2}} G^{N}\left(1+O\left(e^{-N^{1-\varepsilon}}\right)\right), \quad N \rightarrow \infty,
$$

where

$$
C=1-e^{-4 \gamma}, \quad G=e^{\gamma-t}, \quad F=\sinh (\gamma+t)
$$

see [7, so that the constant factor $C$ is known explicitly in the ferroelectric phase.

Antiferroelectric phase region. Here, with parameterization (1.2),

$$
Z_{N}=C F^{N^{2}} \vartheta_{4}(N \omega)\left(1+O\left(N^{-1}\right)\right), \quad N \rightarrow \infty,
$$

where

$$
\omega=\frac{\pi}{2}\left(1+\frac{t}{\gamma}\right), \quad F=\frac{\pi(\sinh (\gamma-t) \sinh (\gamma+t)) \vartheta_{1}^{\prime}(0)}{2 \gamma \vartheta_{1}(\omega)}
$$

and $\vartheta_{1}(z)=\vartheta_{1}(z \mid q), \vartheta_{4}(z)=\vartheta_{4}(z \mid q)$ are the Jacobi theta functions with the elliptic nome $q=e^{-\frac{\pi^{2}}{2 \gamma}}$, see [9]. It is known that the constant factor $C$ does not depend on $t$, so that

$$
C=C(\gamma)>0,
$$

but its exact value is not known.

Disordered phase region. Compare [6], with parameterization (1.3) for some $\varepsilon>0$,

$$
Z_{N}=C F^{N^{2}} N^{\kappa}\left(1+O\left(N^{-\varepsilon}\right)\right), \quad N \rightarrow \infty,
$$

with

$$
\kappa=\frac{1}{12}-\frac{2 \gamma^{2}}{3 \pi(\pi-2 \gamma)}, \quad F=\frac{\pi(\sin (\gamma-t) \sin (\gamma+t))}{2 \gamma \cos \frac{\pi t}{2 \gamma}} .
$$

It is known that the constant factor $C>0$ has the following dependence on $t$ :

$$
C=C_{0}(\gamma)\left(\cos \frac{\pi t}{2 \gamma}\right)^{\kappa},
$$

but the function $C_{0}(\gamma)>0$ is not known. 
Critical line between the antiferroelectric and disordered phase regions. With the parameterization $a=1-x, b=1+x, c=2,|x|<1$, see [5],

$$
(\mathrm{AF}-\mathrm{D}) \quad Z_{N}=C F^{N^{2}} N^{\frac{1}{12}}\left(1+O\left(N^{-1}\right)\right), \quad N \rightarrow \infty
$$

where

$$
F=\frac{\pi\left(1-x^{2}\right)}{2 \cos \frac{\pi x}{2}}
$$

The constant factor $C>0$ has the structure

$$
C=C_{0}\left(\cos \frac{\pi x}{2}\right)^{\frac{1}{12}}
$$

where the universal constant $C_{0}>0$ is not known.

In the last three cases, the structural information (1.20), (1.21), and (1.22) on the constant factors is obtained by combining the results in [9, 6, 5, with the Toda equation. This can also be done in the present situation (see (1.18)), which then leaves us with the determination of the constants $c$ and $d$. In order to compute them, we use the double scaling asymptotics of the partition function as described above.

In [8, Bleher and Liechty rescaled the original weight (1.10) as

$$
w^{o}(x)=w\left(\frac{x}{\alpha-1}\right)=e^{-x}-e^{-r x}, \quad r=\frac{\alpha+1}{\alpha-1}>1
$$

and studied the constants $h_{n}^{o}$ associated with the monic orthogonal polynomials $\left\{p_{n}^{o}(x)\right\}_{n \geq 0}$, satisfying the orthogonality condition

$$
\int_{0}^{\infty} p_{n}^{o}(x) p_{m}^{o}(x) w^{o}(x) d x=h_{n}^{o} \delta_{n m} .
$$

The main technical result in the work [8] is the following asymptotic formula for $h_{N}^{o}$ : As $N \rightarrow \infty$

$$
\ln \left[\frac{h_{N}^{o}}{(N !)^{2}}\right]=-\frac{\zeta(3 / 2)}{2 \sqrt{\pi(r-1)} N^{1 / 2}}+\frac{1}{4 N}+O\left(N^{-3 / 2}\right)
$$

which holds uniformly on any compact subset of the set (1.16). Applying (1.14), this result implies immediately (1.15), in particular it gives the listed explicit expressions for $F$ and $G$. However we cannot take the limit $\alpha \rightarrow \infty$ in (1.23). To overcome this difficulty, in addition to weight (1.10), we will study the related weight

$$
w_{t}(x)=\frac{w(t x)}{2 t}=x e^{-N V(x)}, \quad V(x)=x-\frac{\tau}{t} \ln S(t x), \quad t=\frac{N}{\alpha}=N \tau, \quad S(x)=\frac{\sinh x}{x}
$$

and its associated monic orthogonal polynomials $\left\{p_{n, t}(x)\right\}_{n \geq 0}$, satisfying the orthogonality condition

$$
\int_{0}^{\infty} p_{n, t}(x) p_{m, t}(x) w_{t}(x) d x=h_{n, t} \delta_{n m} .
$$

Noticing that $h_{N}^{o}=(\alpha-1)^{2 N+1} h_{N}$, we will prove the following generalization of (1.23):

Theorem 1.3. As $N \rightarrow \infty$,

$$
\frac{h_{N}}{(N !)^{2}}=\frac{N}{8} \tau^{2 N+2} b^{2} \exp \left[N(l+2)+\frac{v}{N}-\frac{1}{6 N}+\varepsilon_{N}(\tau)\right], \quad \tau=\frac{1}{\alpha}
$$


where $\varepsilon_{N}$ is smooth in the parameter $\tau$ with

$$
\left|\varepsilon_{N}\right| \leq \frac{c}{(N+1)^{2}}, \quad\left|\frac{d \varepsilon_{N}}{d \tau}\right| \leq \frac{c}{(N+1)^{2}}, \quad c>0
$$

and the stated expansion (1.25) is uniform with respect to the parameter $0 \leq \tau \leq 1-\varepsilon<1$. Here, the parameter $b$ is determined implicitly via the equation

$$
b=\frac{4}{1-\tau}+\frac{2 \tau}{(1-\tau) t} I(2 b t), \quad I(z)=-1+\frac{z}{\pi} \int_{0}^{1} \sqrt{\frac{u}{1-u}} \frac{d u}{e^{z u}-1}, \quad z \geq 0,
$$

the parameter $l$ equals

$$
l=4(1-\ln 2)-\frac{b}{2}(1-\tau)-b+2 \ln b-\frac{2 \tau}{t}(1-\ln 2)+\frac{2 \tau}{t} J(2 b t)+\frac{\tau}{t} \ln S(t b)
$$

with

$$
J(z)=\frac{z}{\pi} \int_{0}^{1}\left(\sqrt{\frac{u}{1-u}}-\arctan \sqrt{\frac{u}{1-u}}\right) \frac{d u}{e^{z u}-1}, \quad z \geq 0
$$

and

$$
v=\frac{3}{4 b q(0)}-\frac{q^{\prime}(b)}{4 q^{2}(b)}+\frac{47}{12 b q(b)}, \quad q(z)=\frac{1}{2 \pi} \oint_{\Gamma} \sqrt{\frac{w}{w-b}} \frac{V^{\prime}(w)}{w-z} d w,
$$

where $\Gamma$ is a counter-clockwise oriented contour containing $[0, b] \cup\{z\}$ in its interior such that $V(z)$ is analytic in the interior of $\Gamma$.

The proof of Theorem 1.3 relies on the Riemann-Hilbert approach to the orthogonal polynomials associated with the weight $w_{t}$,

$$
w_{t}(x)=x e^{-N V(x)}, x \geq 0 ; \quad V(z)=z(1-\tau H(t z)), \quad H(z)=\frac{1}{z} \ln \left(\frac{\sinh z}{z}\right)
$$

where the potential $V$ is analytic in the strip

$$
\Delta_{t}=\left\{z \in \mathbb{C}:-\frac{\pi}{t}<\operatorname{Im} z<\frac{\pi}{t}\right\} .
$$

This potential is somewhat close to the class of Laguerre potentials which were considered by Vanlessen in [27]. We use a combination of techniques from [27] and [8] to derive (1.25). The explicit form of $C$ in (1.17) will then follow from an interplay of the Toda equation (1.13) with (1.25) which we combine with (1.14). In particular we use the fact, that, as $\alpha \rightarrow \infty$ and $N$ is fixed, $w_{t}$ has the nontrivial limit

$$
\lim _{\alpha \rightarrow \infty} w_{t}(x)=x e^{-N x}, \quad x \geq 0
$$

and therefore the limiting orthogonal polynomials are classical Laguerre polynomials.

The structure of the rest of the article is as follows. We derive Theorem 1.3 through the RiemannHilbert approach to orthogonal polynomials. This requires the construction of the equilibrium measure as stated in Section 2, i.e. in particular, evaluation of the right endpoint $b$ of its support, its density, and the Lagrange multiplier $l$. Then, following the Deift-Zhou nonlinear steepest descent roadmap, we carry out in Section 3 a sequence of transformations. These will allow us to approximate the solution of the initial Riemann-Hilbert problem by explicit parametrices and by an iterative solution of a singular integral equation. As an application of this analysis, we prove in Section 4 Theorem 1.3. In Section 5. we use the Toda equation to derive formula (1.19) for the constant $C$. Then, in Section 6, we prove the double scaling asymptotics of the partition function. Finally, in Section 7, we prove Theorem 1.2 . 


\section{EQUiLiBRium MEASURE}

2.1. Definition of the equilibrium measure and evaluation of the endpoint of its support. We have rescaled the original weight function $w(x)$ from (1.10) as

$$
w_{t}(x)=\frac{w(t x)}{2 t}=x e^{-N V(x)}, \quad V(z)=z(1-\tau H(t z)), \quad H(z)=\frac{1}{z} \ln \left(\frac{\sinh z}{z}\right), \quad t=N \tau=\frac{N}{\alpha},
$$

hence the associated monic orthogonal polynomials $\left\{p_{n, t}(x)\right\}_{n \geq 0}$ are related to the initial ones in (1.11) via the relations

$$
h_{n}=2 t^{2 n+2} h_{n, t}, \quad p_{n}(x)=t^{n} p_{n, t}\left(\frac{x}{t}\right), \quad n \geq 0 .
$$

Thus, after computing the large $N$ asymptotics of $h_{N, t}$, we can evaluate $\tau_{N}$ from (1.12) via $h_{N}=$ $2 t^{2 N+2} h_{N, t}$.

Notice that we can write the Hankel determinant as

$$
\tau_{N}=\frac{2^{N} t^{N(N+1)}}{N !} \int_{0}^{\infty} \ldots \int_{0}^{\infty}\left(\prod_{j=1}^{N} y_{j}\right) e^{-N \sum_{j=1}^{N} V\left(y_{j}\right)} \prod_{i<j}\left(y_{i}-y_{j}\right)^{2} d y_{1} \cdots d y_{N}
$$

which allows us, with the help of the empirical measure $\nu$ on $[0, \infty)$

$$
\nu(s)=\frac{1}{N} \sum_{k=1}^{N} \delta\left(s-y_{k}\right), \quad \int_{0}^{\infty} d \nu(s)=1,
$$

to express parts of the integrand as

$$
e^{-N \sum_{j=1}^{N} V\left(y_{j}\right)} \prod_{i<j}\left(y_{i}-y_{j}\right)^{2}=e^{-N^{2} H(\nu)},
$$

with the energy functional

$$
H(\nu)=\iint \ln |t-s|^{-1} d \nu(t) d \nu(s)+\int V(s) d \nu(s) .
$$

This observation leads to the expectation that the value of $\tau_{N}$, as $N \rightarrow \infty$, will be concentrated in a vicinity of the global minimum of the functional $H(\nu)$, with $\nu$ varying over

$$
\mathcal{M}^{1}[0, \infty)=\left\{\mu \in \text { Borel measures on }[0, \infty): \int d \mu=1\right\} .
$$

But it is well known (cf. [16, 17]) that the minimization problem

$$
E^{V}=\inf _{\mu \in \mathcal{M}^{1}[0, \infty)}\left[\iint \ln |t-s|^{-1} d \mu(t) d \mu(s)+\int V(s) d \mu(s)\right]
$$

has a unique solution $\mu=\mu^{V} \in \mathcal{M}^{1}[0, \infty)$, the equilibrium measure. We now begin to gather various characteristica of the equilibrium measure:

As the potential $V(z)=z(1-\tau H(t z))$ is convex, the support of the equilibrium measure $\mu^{V}$ consists of a single interval

$$
J=[0, b] \subset \mathbb{R} .
$$


Our first goal is to derive an expansion for the right endpoint $b$, as $N \rightarrow \infty$ for different values of the double scaling parameter $t$. To this end use the $g$-function

$$
g(z)=\int_{J} \ln (z-w) d \mu^{V}(w)=\int_{0}^{b} \ln (z-w) \psi(w) d w, \quad z \in \mathbb{C} \backslash(-\infty, b]
$$

with the principal branch chosen in the logarithm. The equilibrium measure determines the $g$ function by definition, but on the other hand the $g$-function determines the equilibrium measure uniquely through a set of variational conditions:

$$
z \in[0, \infty) \backslash J: g_{+}(z)+g_{-}(z)-V(z)-l \leq 0, \quad z \in J: g_{+}(z)+g_{-}(z)-V(z)-l=0,
$$

where $l \in \mathbb{R}$ is the Lagrange multiplier. The latter equality on the support $J$, leads to an additive Riemann-Hilbert problem for the unknown $g^{\prime}(z)$ which is solved explicitly

$$
g^{\prime}(z)=\frac{1}{2 \pi} \sqrt{\frac{z}{z-b}} \int_{0}^{b} \sqrt{\frac{w}{b-w}} \frac{V^{\prime}(w)}{z-w} d w, \quad z \in \mathbb{C} \backslash[0, b] .
$$

Comparing the large $z$-asymptotics of the last equation with the one obtained from (2.1), we derive the following defining equation on the right endpoint $b$

$$
\frac{1}{2 \pi} \int_{0}^{b} \sqrt{\frac{w}{b-w}} V^{\prime}(w) d w=1 .
$$

Since

$$
V^{\prime}(z)=1-\tau\left(\frac{\cosh (t z)}{\sinh (t z)}-\frac{1}{t z}\right)=1-\tau\left(1-\frac{1}{t z}+\frac{2}{e^{2 t z}-1}\right)
$$

we obtain from (2.3) after the change of variables $w=b u$

$$
b=\frac{4}{1-\tau}+\frac{2 \tau}{(1-\tau) t} I(2 b t), \quad I(z)=-1+\frac{z}{\pi} \int_{0}^{1} \sqrt{\frac{u}{1-u}} \frac{d u}{e^{z u}-1}, \quad z \geq 0
$$

which is equation (1.26) in Theorem 1.3. We will solve the last equation for $b$ iteratively, before that, let us study the asymptotic behavior of $I(z)$ as $z \rightarrow 0$ and $z \rightarrow+\infty$.

Proposition 2.1. The function $I(z)$ is analytic in the strip

$$
\Delta=\{z \in \mathbb{C}:-\pi<\operatorname{Im} z<\pi\}
$$

with

and as $z \rightarrow+\infty$,

$$
I(z)=-\frac{z}{4}+\frac{z^{2}}{32}+O\left(z^{3}\right), \quad z \rightarrow 0,
$$

$$
I(z)=-1+\frac{\zeta(3 / 2)}{2 \sqrt{\pi} z^{1 / 2}}+\frac{3 \zeta(5 / 2)}{8 \sqrt{\pi} z^{3 / 2}}+O\left(z^{-5 / 2}\right) .
$$

Proof. Analyticity of $I(z)$ in $\Delta$ follows immediately from the analyticity of $\frac{z}{e^{z}-1}$ in $\Delta$, hence we are left with the two asymptotic expansions. When $z \rightarrow 0$, we use the asymptotic formula,

$$
\frac{1}{e^{z}-1}=\frac{1}{z}-\frac{1}{2}+\frac{z}{12}+O\left(z^{2}\right), \quad z \rightarrow 0
$$


combined with the integrals,

$$
\int_{0}^{1} \frac{d u}{\sqrt{u(1-u)}}=\pi, \quad \int_{0}^{1} \sqrt{\frac{u}{1-u}} d u=\frac{\pi}{2}, \quad \int_{0}^{1} u \sqrt{\frac{u}{1-u}} d u=\frac{3 \pi}{8}
$$

to obtain

$$
I(z)=-1+\frac{z}{\pi} \int_{0}^{1} \sqrt{\frac{u}{1-u}}\left(\frac{1}{z u}-\frac{1}{2}+\frac{z u}{12}+O\left(z^{2} u^{2}\right)\right) d u=-\frac{z}{4}+\frac{z^{2}}{32}+O\left(z^{3}\right),
$$

which is (2.4). When $z \rightarrow+\infty$, we use the asymptotic formula,

$$
\sqrt{\frac{u}{1-u}}=u^{1 / 2}+\frac{u^{3 / 2}}{2}+O\left(u^{5 / 2}\right), \quad u \rightarrow 0,
$$

and the integrals,

$$
\int_{0}^{\infty} \frac{u^{1 / 2}}{e^{u}-1} d u=\frac{\sqrt{\pi}}{2} \zeta\left(\frac{3}{2}\right), \quad \int_{0}^{\infty} \frac{u^{3 / 2}}{e^{u}-1} d u=\frac{3 \sqrt{\pi}}{4} \zeta\left(\frac{5}{2}\right)
$$

which gives (2.5).

Let us now return to equation (1.26). Since $\alpha>1$, we have that $\tau<1$ and we will in fact assume from now on, that $\tau$ is separated from 1 , so that

$$
0 \leq \tau \leq 1-\varepsilon<1,
$$

where $\varepsilon>0$ is fixed throughout the remainder of this paper. To solve (1.26) for $b$, use iterations

$$
b_{j}=\frac{4}{1-\tau}+\frac{2 \tau}{(1-\tau) t} I\left(2 b_{j-1} t\right), j \geq 1 \quad b_{0}=\frac{4}{1-\tau} .
$$

Consider the mapping

$$
b \stackrel{f}{\mapsto} \frac{4}{1-\tau}+\frac{2 \tau}{(1-\tau) t} I(2 b t)
$$

which satisfies

$$
\frac{d f}{d b}=\frac{4 \tau}{(1-\tau)} I^{\prime}(2 b t) .
$$

From (2.5), after differentiation, we obtain the estimate,

$$
\left|\frac{d f}{d b}\right| \leq \frac{C \tau}{1+t^{3 / 2}}, \quad t>0, \quad b>1,
$$

where $C>0$ is a constant independent of $\tau$ and $t$, i.e. the mapping $f$ is contracting for small $\tau$ in a neighborhood of the point $b_{0}$, and hence $f$ has a fixed point $b$ which can be obtained as a limit of the iterations

$$
b_{j}=f\left(b_{j-1}\right), \quad j \geq 1 .
$$

In addition, we obtain the estimate of the difference $\left|b_{j}-b\right|$ as

$$
\left|b_{j}-b\right| \leq \hat{C}\left(\frac{\tau}{1+t^{3 / 2}}\right)^{j+1}, \hat{C}>0 .
$$

Back to (2.6), we have in the first iteration

$$
b_{1}=\frac{4}{1-\tau}+\frac{2 \tau}{(1-\tau) t} I\left(\frac{8 t}{1-\tau}\right)
$$


and in the second,

$$
b_{2}=\frac{4}{1-\tau}+\frac{2 \tau}{(1-\tau) t} I\left(2 t b_{1}\right)=\frac{4}{1-\tau}+\frac{2 \tau}{(1-\tau) t} I\left(\frac{8 t}{1-\tau}+\frac{4 t \tau}{(1-\tau) t} I\left(\frac{8 t}{1-\tau}\right)\right) .
$$

Combining now (2.8) with (2.7) we obtain

Proposition 2.2. As $N \rightarrow \infty$, the right endpoint $b$ of the equilibrium measure has the asymptotic behavior,

$$
b=\frac{4}{1-\tau}+\frac{2 \tau}{(1-\tau) t} I\left(\frac{8 t}{1-\tau}+\frac{4 \tau}{1-\tau} I\left(\frac{8 t}{1-\tau}\right)\right)+O\left(\left(\frac{\tau}{1+t^{3 / 2}}\right)^{3}\right)
$$

which is uniform with respect to the parameters $0 \leq \tau \leq 1-\varepsilon<1$ and $t \geq 0$.

At this point we would like to collect some facts on the density $\psi(x), x \in[0, b]$ of the equilibrium measure as introduced in (2.1).

2.2. Evaluation of the density of the equilibrium measure. We use [17] and [27], more precisely the identities

with (compare (1.29)

$$
\psi(x)=\frac{1}{2 \pi} \sqrt{\frac{b-x}{x}} q(x), \quad x \in[0, b]
$$

$$
q(z)=\frac{1}{2 \pi i} \oint_{\Gamma} \sqrt{\frac{w}{w-b}} \frac{V^{\prime}(w)}{w-z} d w
$$

where $\Gamma$ is a counter-clockwise oriented contour containing $[0, b] \cup\{z\}$ in its interior such that $V(z)$ is analytic in the interior of $\Gamma$. Replacing $V^{\prime}(w)$ by

$$
V^{\prime}(z)=1-\tau k(t z), \quad k(z)=\frac{\cosh z}{\sinh z}-\frac{1}{z}
$$

we obtain after applying residue theorem

$$
q(z)=1+\tau s(z, t), \quad s(z, t)=-\frac{1}{2 \pi i} \oint_{\Gamma} \sqrt{\frac{w}{w-b}} \frac{k(t w)}{w-z} d w .
$$

The properties of the function $s(z, t)$ will be important for us. They are described as follows:

Proposition 2.3. The function $s(z, t)$ is real analytic on the set $[0, b] \times \mathbb{R}$. As $t \rightarrow 0$,

$$
s(z, t)=-\frac{t}{3}\left(z+\frac{b}{2}\right)+\frac{t^{3}}{45}\left(z^{3}+\frac{b}{2} z^{2}+\frac{3 b^{2}}{8} z+\frac{5 b^{3}}{16}\right)+O\left(t^{5}\right),
$$

and as $t \rightarrow \infty$,

$$
s(z, t)=-1+O\left(t^{-1 / 2}\right), \quad \frac{\partial s(z, t)}{\partial z}=O\left(t^{-1 / 2}\right),
$$

uniformly in $z \in[0, b]$.

Proof. The integrand in (2.10) is analytic with respect to $(z, t) \in[0, b] \times \mathbb{R}$, hence $s(z, t)$ is analytic as well and $s(z, t)$ is real-valued since the contour $\Gamma$ can be deformed to the interval $[0, b]$ covered twice. Let us now derive the asymptotic formulae for $s(z, t)$. When $t \rightarrow 0$, use

$$
k(z)=\frac{z}{3}-\frac{z^{3}}{45}+O\left(z^{5}\right), \quad z \rightarrow 0
$$


and obtain, as $t \rightarrow 0$,

$$
s(z, t)=-\frac{1}{2 \pi i} \oint_{\Gamma} \sqrt{\frac{w}{w-b}} \frac{t w}{3} \frac{d w}{w-z}+\frac{1}{2 \pi i} \oint_{\Gamma} \sqrt{\frac{w}{w-b}} \frac{(t w)^{3}}{45} \frac{d w}{w-z}+O\left(t^{5}\right),
$$

hence equation (2.11) follows from residue theorem, noticing that

$$
\begin{aligned}
\sqrt{\frac{w}{w-b}} \frac{1}{w-z}= & 1+\frac{1}{w}\left(z+\frac{b}{2}\right)+\frac{1}{w^{2}}\left(z^{2}+\frac{b}{2} z+\frac{3 b^{2}}{8}\right)+\frac{1}{w^{3}}\left(z^{3}+\frac{b}{2} z^{2}\right. \\
& \left.+\frac{3 b^{2}}{8} z+\frac{5 b^{3}}{16}\right)+O\left(w^{-4}\right), \quad|w| \rightarrow \infty .
\end{aligned}
$$

For the expansions (2.12), we rewrite $s(z, t)$ as

$$
s(z, t)=-\frac{1}{2 \pi i} \oint_{\Gamma} \sqrt{\frac{w}{w-b}}\left(1-\frac{1}{t w}+\frac{2}{e^{2 t w}-1}\right) \frac{d w}{w-z}=-1-\frac{1}{2 \pi i} \oint_{\Gamma} \sqrt{\frac{w}{w-b}} \frac{2}{e^{2 t w}-1} \frac{d w}{w-z},
$$

where the last equality follows once more from residue theorem. After the change of variables $u=2 t w$,

$$
s(z, t)=-1-\frac{1}{2 \pi i} \oint_{2 t \Gamma} \sqrt{\frac{u}{u-2 t b}} \frac{2}{e^{u}-1} \frac{d u}{u-2 t z}
$$

and we now choose the contour $2 t \Gamma$ to be a long "stadium", so that it consists of two parallel segments, $\{u=x \pm i, 0 \leq x \leq 2 t b\}$ and two semicircles of radius 1 , around the points $u=0$ and $u=2 t b$. With this choice

$$
\left|\sqrt{\frac{u}{u-2 t b}} \frac{2}{e^{u}-1} \frac{1}{u-2 t z}\right| \leq \frac{C}{\sqrt{1+t}}, \quad u \in 2 t \Gamma,
$$

and we obtain the first estimation in (2.12). For the second, we differentiate with respect to $z$, i.e.

$$
\frac{\partial s(z, t)}{\partial z}=-\frac{1}{2 \pi i} \oint_{\Gamma} \sqrt{\frac{w}{w-b}} \frac{2}{e^{2 t w}-1} \frac{d w}{(w-z)^{2}},
$$

and change again variables $u=2 t w$. Estimating the latter integral from above, we obtain the remaining estimation in (2.12).

We can now combine (2.10) with Proposition 2.3 and deduce

$$
\sup _{0 \leq y \leq b}|q(y)-1+\tau|=O\left(\frac{\tau}{\sqrt{1+t}}\right), \quad \sup _{0 \leq y \leq b}\left|q^{\prime}(y)\right|=O\left(\frac{\tau}{\sqrt{1+t}}\right),
$$

which is uniform with respect to the parameters $0 \leq \tau \leq 1-\varepsilon<1$ and $t \geq 0$.

We are left with the computation of the Lagrange multiplier.

2.3. Evaluation of the Lagrange multiplier. We will compute the multiplier $l$ via (2.2),

$$
l=2 g(b)-V(b),
$$

in other words, we have to compute two quantities. For $g(b)$, use the formula

$$
g(b)=\ln b-\int_{b}^{\infty}\left(\omega(z)-\frac{1}{z}\right) d z
$$


which involves the resolvent $\omega(z) \equiv g^{\prime}(z)$ and which can be derived immediately from the expansion

$$
g^{\prime}(z)=\int_{0}^{b} \frac{d \mu^{V}(w)}{z-w}=\frac{1}{z}+O\left(z^{-2}\right), \quad z \rightarrow \infty .
$$

As we have already seen,

$$
\omega(z)=\frac{1}{2 \pi} \sqrt{\frac{z-b}{z}} \int_{0}^{b} \sqrt{\frac{w}{b-w}} \frac{V^{\prime}(w)}{z-w} d w,
$$

and the latter equality can be rewritten as (cf. [17])

$$
\omega(z)=\frac{V^{\prime}(z)}{2}-\sqrt{\frac{z-b}{z}} \frac{q(z)}{2}, \quad z \in \mathbb{C} \backslash[0, b] .
$$

Back to (2.15), this implies

$$
g(b)=\ln b-\frac{1}{2} \int_{b}^{\infty}\left(V^{\prime}(z)-\sqrt{\frac{z-b}{z}} q(z)-\frac{2}{z}\right) d z .
$$

Deforming the contour of integration in (2.13) and evaluating the residue at $w=z$, we have

$$
s(z, t)=-1-\sqrt{\frac{z}{z-b}} \frac{2}{e^{2 t z}-1}-\frac{1}{\pi} \int_{0}^{b} \sqrt{\frac{w}{b-w}} \frac{2}{e^{2 t w}-1} \frac{d w}{w-z}
$$

and with

$$
V^{\prime}(z)=1-\tau\left(1-\frac{1}{t z}+\frac{2}{e^{2 t z}-1}\right)
$$

therefore

$$
\begin{aligned}
g(b)= & \ln b-\frac{1}{2} \int_{b}^{\infty}\left[1-\tau+\frac{\tau}{t z}-\frac{2 \tau}{e^{2 t z}-1}-\sqrt{\frac{z-b}{z}}\left(1-\tau-\sqrt{\frac{z}{z-b}} \frac{2 \tau}{e^{2 t z}-1}\right.\right. \\
& \left.\left.-\frac{\tau}{\pi} \int_{0}^{b} \sqrt{\frac{w}{b-w}} \frac{2}{e^{2 t w}-1} \frac{d w}{w-z}\right)-\frac{2}{z}\right] d z,
\end{aligned}
$$

or after simplifications,

$$
\begin{aligned}
g(b)= & \ln b-\frac{1}{2} \int_{b}^{\infty}\left[(1-\tau)\left(1-\sqrt{\frac{z-b}{z}}\right)+\frac{\tau}{t z}-\frac{2}{z}+\sqrt{\frac{z-b}{z}} \frac{\tau}{\pi}\right. \\
& \left.\times \int_{0}^{b} \sqrt{\frac{w}{b-w}} \frac{2}{e^{2 t w}-1} \frac{d w}{w-z}\right] d z .
\end{aligned}
$$

At this point we use (1.26) and write

$$
-\frac{\tau}{z \pi} \int_{0}^{b} \sqrt{\frac{w}{b-w}} \frac{2}{e^{2 t w}-1} d w=\frac{1}{z}\left(2-\frac{b}{2}(1-\tau)-\frac{\tau}{t}\right),
$$

hence (2.16) reads as

$$
g(b)=\ln b+I_{1}+I_{2},
$$


where

$$
I_{1}=-\frac{1}{2} \int_{b}^{\infty}\left[(1-\tau)\left(1-\sqrt{\frac{z-b}{z}}\right)+\frac{\tau}{t z}-\frac{2}{z}+\frac{1}{z} \sqrt{\frac{z-b}{z}}\left(2-\frac{b}{2}(1-\tau)-\frac{\tau}{t}\right)\right] d z,
$$

and

$$
I_{2}=\frac{\tau}{\pi} \int_{b}^{\infty} \sqrt{\frac{z-b}{z}} \frac{1}{z}\left[\int_{0}^{b} \sqrt{\frac{w}{b-w}} \frac{w d w}{\left(e^{2 t w}-1\right)(z-w)}\right] d z .
$$

The term $I_{1}$ is calculated explicitly,

$$
I_{1}=2(1-\ln 2)-\frac{b}{4}(1-\tau)-\frac{\tau}{t}(1-\ln 2)=2-2 \ln 2-\frac{b}{4}(1-\tau)-\frac{1}{N}+\frac{\ln 2}{N},
$$

and in $I_{2}$ we change the order of integration: Since

$$
\int_{b}^{\infty} \sqrt{\frac{z-b}{z}} \frac{d z}{z(z-w)}=\frac{2}{w}\left(1-\sqrt{\frac{b-w}{w}} \arctan \sqrt{\frac{w}{b-w}}\right), \quad 0<w<b,
$$

we obtain

$$
I_{2}=\frac{2 \tau}{\pi} \int_{0}^{b}\left(\sqrt{\frac{w}{b-w}}-\arctan \sqrt{\frac{w}{b-w}}\right) \frac{d w}{e^{2 t w}-1}=\frac{\tau}{t} J(2 b t)
$$

with (compare (1.28)

$$
J(z)=\frac{z}{\pi} \int_{0}^{1}\left(\sqrt{\frac{u}{1-u}}-\arctan \sqrt{\frac{u}{1-u}}\right) \frac{d u}{e^{z u}-1} .
$$

Some important properties of the function $J(z)$ are summarized below.

Proposition 2.4. The function $J(z)$ is analytic in the horizontal strip $\Delta$. As $z \rightarrow 0$,

$$
J(z)=1-\ln 2-\frac{z}{8}+\frac{7 z^{2}}{384}+O\left(z^{3}\right)
$$

and as $z \rightarrow+\infty$,

$$
J(z)=\frac{\zeta(5 / 2)}{4 \sqrt{\pi} z^{3 / 2}}+\frac{9 \zeta(7 / 2)}{16 \sqrt{\pi} z^{5 / 2}}+O\left(z^{-7 / 2}\right) .
$$

Proof. Our reasoning is almost identical to the one given in the proof of Proposition 2.1, in particular analyticity follows from the analyticity of $\frac{z}{e^{z}-1}$ in $\Delta$. When $z \rightarrow 0$, we use the integrals

$$
\begin{gathered}
\int_{0}^{1}\left(\sqrt{\frac{u}{1-u}}-\arctan \sqrt{\frac{u}{1-u}}\right) \frac{d u}{u}=\pi(1-\ln 2) \\
\int_{0}^{1}\left(\sqrt{\frac{u}{1-u}}-\arctan \sqrt{\frac{u}{1-u}}\right) d u=\frac{\pi}{4}, \quad \int_{0}^{1}\left(\sqrt{\frac{u}{1-u}}-\arctan \sqrt{\frac{u}{1-u}}\right) u d u=\frac{7 \pi}{32}
\end{gathered}
$$


and obtain

$$
\begin{aligned}
J(z) & =\frac{z}{\pi} \int_{0}^{1}\left(\sqrt{\frac{u}{1-u}}-\arctan \sqrt{\frac{u}{1-u}}\right)\left(\frac{1}{z u}-\frac{1}{2}+\frac{z u}{12}+O\left(z^{2} u^{2}\right)\right) d u \\
& =1-\ln 2-\frac{z}{8}+\frac{7 z^{2}}{384}+O\left(z^{3}\right),
\end{aligned}
$$

which is (2.19). When $z \rightarrow+\infty$, we use the expansion

$$
\sqrt{\frac{u}{1-u}}-\arctan \sqrt{\frac{u}{1-u}}=\frac{u^{3 / 2}}{3}+\frac{3}{10} u^{5 / 2}+O\left(u^{7 / 2}\right), \quad u \rightarrow 0,
$$

and the integrals,

$$
\int_{0}^{\infty} \frac{u^{3 / 2}}{e^{u}-1} d u=\frac{3 \sqrt{\pi}}{4} \zeta\left(\frac{5}{2}\right), \quad \int_{0}^{\infty} \frac{u^{5 / 2}}{e^{u}-1} d u=\frac{15 \sqrt{\pi}}{8} \zeta\left(\frac{7}{2}\right),
$$

which gives (2.20).

In the end we go back to (2.17) and summarize

$$
g(b)=\ln b+2(1-\ln 2)-\frac{b}{4}(1-\tau)-\frac{\tau}{t}(1-\ln 2)+\frac{\tau}{t} J(2 b t),
$$

which, combined with $V(b)=b-\frac{\tau}{t} \ln S(b t)$, gives equation (1.27), i.e.

$$
l=4(1-\ln 2)-\frac{b}{2}(1-\tau)-b+2 \ln b-\frac{2 \tau}{t}(1-\ln 2)+\frac{2 \tau}{t} J(2 b t)+\frac{\tau}{t} \ln S(b t) .
$$

At this point we can begin the asymptotical analysis.

\section{Riemann-Hilbert apPROACH}

3.1. Riemann-Hilbert characterization for orthogonal polynomials. We will solve the FokasIts-Kitaev [21] Riemann-Hilbert problem (RHP) for orthogonal polynomials asymptotically: this problem requires the construction of a $2 \times 2$ piecewise analytic matrix-valued function $Y(z)=$ $Y^{(n)}(z)$ such that

- $Y^{(n)}(z)$ is analytic for $z \in \mathbb{C} \backslash[0, \infty)$

- If we orient the half ray $[0, \infty)$ from left to right, the limiting values of $Y^{(n)}(z)$ from either side are related via the equation

$$
Y_{+}^{(n)}(z)=Y_{-}^{(n)}(z)\left(\begin{array}{cc}
1 & w_{t}(z) \\
0 & 1
\end{array}\right), \quad z \in[0, \infty)
$$

- At the endpoint $z=0, Y(z)$ remains bounded, i.e.

$$
Y(z)=O(1), \quad z \rightarrow 0, \quad z \in \mathbb{C} \backslash[0, \infty)
$$

- As $z \rightarrow \infty$, we have

$$
Y^{(n)}(z)=\left(I+O\left(z^{-1}\right)\right) z^{n \sigma_{3}}, \quad \sigma_{3}=\left(\begin{array}{cc}
1 & 0 \\
0 & -1
\end{array}\right) .
$$

The unique solution $Y^{(n)}(z)$ to the latter problem equals

$$
Y^{(n)}(z)=\left(\begin{array}{cc}
p_{n, t}(z) & \frac{1}{2 \pi i} \int_{0}^{\infty} p_{n, t}(s)(s) w_{t}(s) \frac{d s}{s-z} \\
\gamma_{n-1} p_{n-1, t}(z) & \frac{\gamma_{n-1}}{2 \pi i} \int_{0}^{\infty} p_{n-1, t}(s) w_{t}(s) \frac{d s}{s-z}
\end{array}\right)
$$


where $p_{n, t}(z)=z^{n}+\ldots$ is precisely the $n^{\text {th }}$ monic orthogonal polynomial subject to the measure $d \mu(s)=w_{t}(s) d s$ supported on the half-ray $[0, \infty)$. Moreover,

$$
\gamma_{n}=-\frac{2 \pi i}{h_{n, t}}, \quad h_{n, t}=\int_{0}^{\infty} p_{n, t}^{2}(s) d \mu(s),
$$

and in addition, $Y^{(n)}(z) z^{-n \sigma_{3}}$ has a full asymptotic expansion near infinity:

$$
Y^{(n)}(z) z^{-n \sigma_{3}}=I+\frac{Y_{1}^{(n)}}{z}+O\left(z^{-2}\right), \quad z \rightarrow \infty, \quad Y_{k}^{(n)}=\left(Y_{k}^{(n)}\right)_{i j} .
$$

This expansion connects to the normalizing constants via

$$
h_{n, t}=-2 \pi i\left(Y_{1}^{(n)}\right)_{12} .
$$

Since we want to compute the large $N$ asymptotics of $h_{N, t}$, we will need to solve the latter RHP for $Y(z)=Y^{(N)}(z)$. Such an asymptotic solution can be derived by applying the Deift-Zhou nonlinear steepest descent method [18 paired with techniques which have been developed in [17, 27] and 8. In short, we will approximate the solution $Y(z)$ with the help of solutions of certain RiemannHilbert model problems. The necessary steps are worked out in the subsections below.

3.2. First transformation of the RHP - normalization. Recall (2.1) and introduce

$$
Y(z)=\exp \left(\frac{N l}{2} \sigma_{3}\right) T(z) \exp \left(N\left(g(z)-\frac{l}{2}\right) \sigma_{3}\right), \quad z \in \mathbb{C} \backslash \mathbb{R} .
$$

The analytical properties of $T(z)$ are summarized in the following:

- $T(z)$ is analytic for $z \in \mathbb{C} \backslash[0, \infty)$

- From the jump properties of $g(z)$, compare (2.2), we get that

$$
T_{+}(z)=T_{-}(z)\left(\begin{array}{cc}
e^{-N\left(g_{+}-g_{-}\right)} & z \\
0 & e^{N\left(g_{+}-g_{-}\right)}
\end{array}\right), \quad z \in[0, b]
$$

and

$$
T_{+}(z)=T_{-}(z)\left(\begin{array}{cc}
1 & z e^{N\left(g_{+}+g_{-}-V-l\right)} \\
0 & 1
\end{array}\right), \quad z \in[0, \infty) \backslash[0, b] .
$$

- As $z \rightarrow 0$ and $z \in \mathbb{C} \backslash[0, \infty)$, the function $T(z)$ is bounded,

$$
T(z)=O(1), \quad z \rightarrow 0, \quad z \in \mathbb{C} \backslash[0, \infty)
$$

- At infinity, the transformed function $T(z)$ is now normalized as

$$
T(z)=I+O\left(z^{-1}\right), \quad z \rightarrow \infty .
$$

Consider the jumps (3.3) and (3.4): First, by the Euler Lagrange variational condition (2.2),

$$
g_{+}(z)+g_{-}(z)-V(z)-l<0, \quad z \in[0, \infty) \backslash[0, b],
$$

hence for $z \in(b+\eta, \infty), \eta>0$ fixed,

$$
\left(\begin{array}{cc}
1 & z e^{N\left(g_{+}+g_{-}-V-l\right)} \\
0 & 1
\end{array}\right) \longrightarrow I, \quad N \rightarrow \infty
$$

where the stated convergence is exponentially fast. Secondly for $[0, b]$ : Since $g_{-}(z)=V(z)-g_{+}(z)+$ $l, z \in[0, b]$ the function

$$
G(z)=g_{+}(z)-g_{-}(z)=2 g_{+}(z)-V(z)-l
$$


can be analytically continued in a (in general $t$-dependent) neighborhood of the line segment $[0, b]$ into the upper halfplane. Here we use in particular that $V(z)$ is analytic in the strip $\Delta_{t}$. But since

$$
G(z)=2 \pi i \int_{z}^{b} \psi(w) d w, \quad z \in[0, b]
$$

and therefore

$$
\left.\frac{d}{d y} G(z+i y)\right|_{y=0}=2 \pi \psi(z)>0, \quad z \in(0, b),
$$

we see that the stated (local) continuation of $G(z)$ into the upper half-plane satisfies

$$
\operatorname{Re} G(z)>0 \text { for } \operatorname{Im} z>0 \text {. }
$$

In the lower halfplane the argument is similar:

$$
G(z)=-2 g_{-}(z)+V(z)+l
$$

admits analytical (in general into a $t$-dependent neighborhood) continuation into the lower halfplane so that

$$
\operatorname{Re} G(z)<0 \text { for } \operatorname{Im} z<0 \text {. }
$$

The continuations motivate the use of the matrix factorization

$$
\begin{aligned}
\left(\begin{array}{cc}
e^{-N\left(g_{+}(z)-g_{-}(z)\right)} & z \\
0 & e^{N\left(g_{+}(z)-g_{-}(z)\right)}
\end{array}\right) & =\left(\begin{array}{cc}
1 & 0 \\
\frac{1}{z} e^{N G(z)} & 1
\end{array}\right)\left(\begin{array}{cc}
0 & z \\
-\frac{1}{z} & 0
\end{array}\right)\left(\begin{array}{cc}
1 & 0 \\
\frac{1}{z} e^{-N G(z)} & 1
\end{array}\right) \\
& =S_{L_{1}} S_{P} S_{L_{2}}, \quad z \in(0, b),
\end{aligned}
$$

and thus the second transformation of the RHP.

3.3. Second transformation of the RHP - opening of lenses. We let $\mathcal{L}^{ \pm}$denote the upper (lower) lens, shown in Figure 4. Define

$$
S(z)= \begin{cases}T(z) S_{L_{2}}^{-1}, & z \in \mathcal{L}^{+}, \\ T(z) S_{L_{1}}, & z \in \mathcal{L}^{-}, \\ T(z), & \text { else, }\end{cases}
$$

so that $S(z)$ solves the following RHP

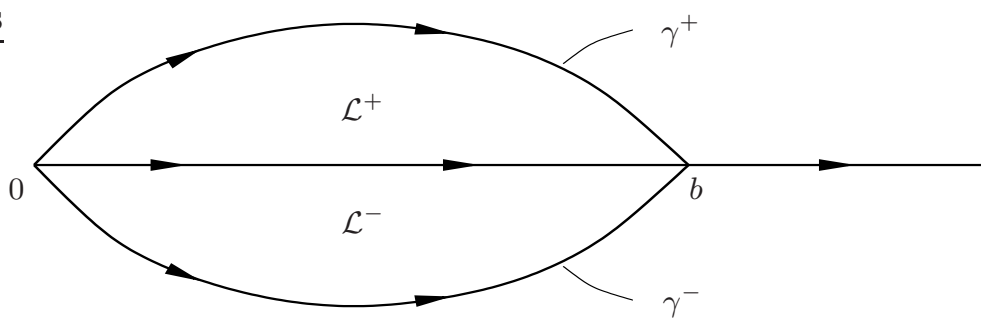

FIGURE 4. The second transformation - opening of lenses

- $S(z)$ is analytic for $z \in \mathbb{C} \backslash([0, \infty) \cup \Gamma)$, with $\Gamma=\gamma^{+} \cup \gamma^{-}$

- The jumps, with orientation fixed as in Figure 4, are as follows

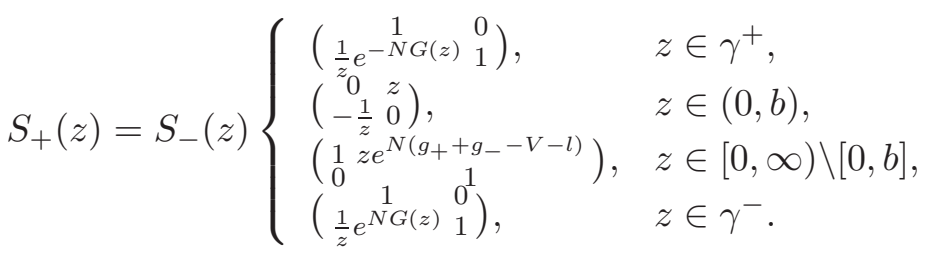


- For the behavior at the origin, we see from the behavior of $T(z)$ and (3.7), that

$$
S(z)=O(1), \quad z \rightarrow 0, z \in \mathbb{C} \backslash\left(\mathcal{L}^{+} \cup \mathcal{L}^{-}\right)
$$

and

$$
S(z)=O\left(z^{-1}\right), \quad z \rightarrow 0, z \in \mathcal{L}^{+} \cup \mathcal{L}^{-} .
$$

- As $z \rightarrow \infty$, we have $S(z)=I+O\left(z^{-1}\right)$.

Recalling (3.5) and (3.6) as well as the behavior of the jump matrix on the infinite ray $(b, \infty)$ we expect (and justify rigorously below) that as $N \rightarrow \infty, S(z)$ converges to a solution of a RHP, in which the only jump is on the line segment $(0, b)$. In more detail, this model RHP reads as follows.

3.4. The model RHP. Find a piecewise analytic $2 \times 2$ matrix valued function $M(z)$ such that

- $M(z)$ is analytic for $z \in \mathbb{C} \backslash[0, b]$

- Along $(0, b)$, we have the boundary relation

$$
M_{+}(z)=M_{-}(z)\left(\begin{array}{cc}
0 & z \\
-\frac{1}{z} & 0
\end{array}\right), \quad z \in(0, b)
$$

- The function $M(z)$ is square integrable on $[0, b]$

- As $z \rightarrow \infty$, the function is normalized as

$$
M(z)=I+O\left(z^{-1}\right)
$$

We compute a solution to this problem by introducing

$$
N(z)=M(z) \mathcal{D}(z)^{\sigma_{3}}, \quad z \in \mathbb{C} \backslash[0, b]
$$

where the scalar Szegö function $\mathcal{D}(z)$ satisfies

$$
\mathcal{D}_{+}(z) \mathcal{D}_{-}(z)=z, \quad z \in[0, b] .
$$

Such a function indeed exists, namely

$$
\mathcal{D}(z)=\exp \left[\frac{\sqrt{z(z-b)}}{2 \pi i} \int_{0}^{b} \frac{\ln w}{\sqrt{w(w-b)}+} \frac{d w}{w-z}\right]=\sqrt{\frac{b z}{2}}\left(z-\frac{b}{2}+\sqrt{z(z-b)}\right)^{-1 / 2}
$$

where we choose principal branches for all fractional power functions. The latter choice of $\mathcal{D}(z)$ transforms the original model problem to a RHP for $N(z)$ with jump

$$
N_{+}(z)=N_{-}(z)\left(\begin{array}{cc}
0 & 1 \\
-1 & 0
\end{array}\right), \quad z \in[0, b]
$$

which is solved via diagonalization. Noticing further that

we obtain

$$
\mathcal{D}(z)=\frac{\sqrt{b}}{2}\left(1+\frac{b}{4 z}+\frac{b^{2}}{8 z^{2}}+O\left(z^{-3}\right)\right), \quad z \rightarrow \infty
$$

$$
M(z)=\left(\frac{\sqrt{b}}{2}\right)^{\sigma_{3}} \frac{1}{2}\left(\begin{array}{cc}
\delta+\delta^{-1} & i\left(\delta-\delta^{-1}\right) \\
-i\left(\delta-\delta^{-1}\right) & \delta+\delta^{-1}
\end{array}\right) \mathcal{D}^{-\sigma_{3}}(z)
$$

with

$$
\delta(z)=\left(\frac{z}{z-b}\right)^{1 / 4}
$$

defined on $\mathbb{C} \backslash[0, b]$ with its branch such that $\left(\frac{z}{z-b}\right)^{1 / 4} \rightarrow 1$ as $z \rightarrow+\infty$, arg $z=0$. Before moving on, we note for future purposes that

$$
M(z)=I+\frac{b}{4 z}\left(\begin{array}{cc}
-1 & \frac{i b}{4} \\
\frac{4}{i b} & 1
\end{array}\right)+O\left(z^{-2}\right), \quad z \rightarrow \infty .
$$


3.5. Construction of a parametrix at $z=b$. For a small neighborhood $\mathcal{U}$ of the point $b$, observe that

$$
G(z)=2 g(z)-V(z)-l=\int_{z}^{b} \sqrt{\frac{w-b}{w}} q(w) d w=-\frac{2}{3} h_{1}(z)(z-b)^{3 / 2}, \quad z \in \mathcal{U} \cap \gamma^{+}
$$

where $h_{1}(z)$ is an analytic function in $\mathcal{U}$ such that

$$
h_{1}(z)=\frac{q(b)}{\sqrt{b}}\left[1+\frac{3}{5}\left(\frac{q^{\prime}(b)}{q(b)}-\frac{1}{2 b}\right)(z-b)+O((z-b))^{2}\right], \quad z \rightarrow b,
$$

and the function $(z-b)^{3 / 2}$ is defined for $z \in \mathbb{C} \backslash(-\infty, b]$ with

$$
(z-b)^{3 / 2}>0 \text { if } z>b .
$$

Similarly, with the same choice of branches,

$$
G(z)=-2 g(z)+V(z)+l=\frac{2}{3} a(z)(z-b)^{3 / 2}, \quad z \in \mathcal{U} \cap \gamma^{-}
$$

and

$$
g_{+}(z)+g_{-}(z)-V(z)-l=-\int_{b}^{z} \sqrt{\frac{w-b}{w}} q(w) d w=-\frac{2}{3} a(z)(z-b)^{3 / 2}, \quad z \in \mathcal{U} \cap(b, \infty) .
$$

The expansions motivate the construction of the parametrix in terms of the Airy function $\operatorname{Ai}(\zeta)$. This construction has appeared frequently in the nonlinear-steepest descent literature and we will follow here the notation of [5]: define for $\zeta \in \mathbb{C}$

$$
A_{0}(\zeta)=\left(\begin{array}{cc}
\frac{d}{d \zeta} \operatorname{Ai}(\zeta) & e^{i \frac{\pi}{3}} \frac{d}{d \zeta} \operatorname{Ai}\left(e^{-i \frac{2 \pi}{3}} \zeta\right) \\
\operatorname{Ai}(\zeta) & e^{i \frac{\pi}{3}} \operatorname{Ai}\left(e^{-i \frac{\pi}{3}} \zeta\right)
\end{array}\right) .
$$

With this, introduce the "bare parametrix"

$$
A^{R H}(\zeta)= \begin{cases}A_{0}(\zeta), & \arg \zeta \in\left(0, \frac{2 \pi}{3}\right), \\
A_{0}(\zeta)\left(\begin{array}{cc}
1 & 0 \\
-1 & 1
\end{array}\right), & \arg \zeta \in\left(\frac{2 \pi}{3}, \pi\right), \\
A_{0}(\zeta)\left(\begin{array}{cc}
1 & -1 \\
0 & 1
\end{array}\right), & \arg \zeta \in\left(-\frac{2 \pi}{3}, 0\right), \\
A_{0}(\zeta)\left(\begin{array}{cc}
0 & -1 \\
1 & 1
\end{array}\right), & \arg \zeta \in\left(-\pi,-\frac{2 \pi}{3}\right) .\end{cases}
$$

which solves the RHP depicted in Figure 5

- $A^{R H}(\zeta)$ is analytic for $\zeta \in \mathbb{C} \backslash\left\{\arg \zeta=-\frac{2 \pi}{3}, 0, \frac{2 \pi}{3}, \pi\right\}$

- We have jumps as sketched in Figure 5

$$
\begin{aligned}
& A_{+}^{R H}(\zeta)=A_{-}^{R H}(\zeta)\left(\begin{array}{cc}
1 & 0 \\
-1 & 1
\end{array}\right), \quad \arg \zeta=\mp \frac{2 \pi}{3} \\
& A_{+}^{R H}(\zeta)=A_{-}^{R H}(\zeta)\left(\begin{array}{cc}
1 & 1 \\
0 & 1
\end{array}\right), \quad \arg \zeta=0 \\
& A_{+}^{R H}(\zeta)=A_{-}^{R H}(\zeta)\left(\begin{array}{cc}
0 & -1 \\
1 & 0
\end{array}\right), \quad \arg \zeta=\pi
\end{aligned}
$$




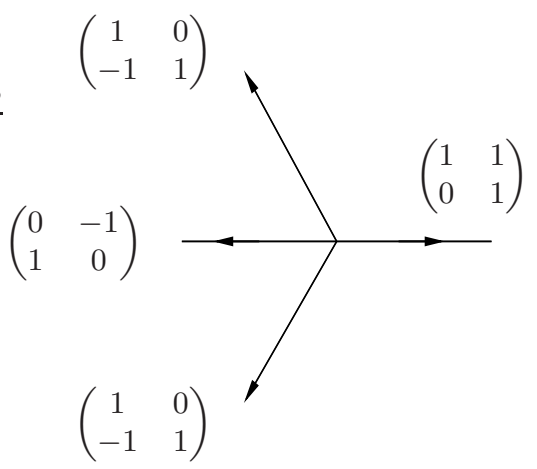

Figure 5. The model RHP near $z=b$ which can be solved explicitly using Airy functions

- From the asymptotics of the Airy function (cf. [4])

$$
\begin{aligned}
A^{R H}(\zeta)= & \frac{\zeta^{\sigma_{3} / 4}}{2 \sqrt{\pi}}\left(\begin{array}{cc}
-1 & i \\
1 & i
\end{array}\right)\left[I+\frac{1}{48 \zeta^{3 / 2}}\left(\begin{array}{cc}
1 & 6 i \\
6 i & -1
\end{array}\right)+\frac{35}{4608 \zeta^{6 / 2}}\left(\begin{array}{cc}
-1 & 12 i \\
-12 i & -1
\end{array}\right)\right. \\
& \left.+O\left(\zeta^{-9 / 2}\right)\right] e^{-\frac{2}{3} \zeta^{3 / 2} \sigma_{3}} .
\end{aligned}
$$

In order to construct the local parametrix to the solution of the $S$-RHP near $z=b$, we first define

$$
\zeta(z)=\left(\frac{3 N}{4}\right)^{2 / 3}(-2 g(z)+V(z)+l)^{2 / 3}, \quad|z-b|<r .
$$

This change of variables is locally conformal, since

$$
\zeta(z)=\left(\frac{N q(b)}{2 \sqrt{b}}\right)^{2 / 3}(z-b)\left[1+\frac{2}{5}\left(\frac{q^{\prime}(b)}{q(b)}-\frac{1}{2 b}\right)(z-b)+O\left((z-b)^{2}\right)\right], \quad|z-b|<r .
$$

Secondly, it allows us to define the right parametrix $U(z)$ near $z=b$ by

$$
U(z)=B_{r}(z)(-i \sqrt{\pi}) A^{R H}(\zeta(z)) e^{\frac{2}{3} \zeta^{3 / 2}(z) \sigma_{3}} z^{-\sigma_{3} / 2}, \quad|z-b|<r
$$

which involves the multiplier

$$
\begin{aligned}
B_{r}(z)= & M(z) z^{\sigma_{3} / 2}\left(\begin{array}{cc}
-i & i \\
1 & 1
\end{array}\right) \zeta^{-\sigma_{3} / 4}(z)=\left(\frac{\sqrt{b}}{2}\right)^{\sigma_{3}}\left(\begin{array}{cc}
-i & i \\
1 & 1
\end{array}\right)\left(\zeta(z) \frac{z}{z-b}\right)^{-\sigma_{3} / 4} \\
& \times \zeta^{\sigma_{3} / 4}(z) \frac{i}{2}\left(\begin{array}{cc}
1 & -i \\
-1 & -i
\end{array}\right) \mathcal{D}^{-\sigma_{3}}(z) z^{\sigma_{3} / 2}\left(\begin{array}{cc}
-i & i \\
1 & 1
\end{array}\right) \zeta^{-\sigma_{3} / 4}(z) .
\end{aligned}
$$

Notice that $B_{r}(z)$ is analytic in a neighborhood of $z=b$, since for $z \in(b-r, b)$

$$
\begin{aligned}
\left(B_{r}(z)\right)_{+} & =M_{+}(z) z^{\sigma_{3} / 2}\left(\begin{array}{cc}
-i & i \\
1 & 1
\end{array}\right) \zeta_{+}^{-\sigma_{3} / 4}(z) \\
& =M_{-}(z)\left(\begin{array}{cc}
0 & z \\
-\frac{1}{z} & 0
\end{array}\right) z^{\sigma_{3} / 2}\left(\begin{array}{cc}
-i & i \\
1 & 1
\end{array}\right) \zeta_{-}^{-\sigma_{3} / 4}(z) e^{-i \frac{\pi}{2} \sigma_{3}}=\left(B_{r}(z)\right)_{-},
\end{aligned}
$$

and by a direct computation

$$
B_{r}(b)=\left(\frac{\sqrt{b}}{2}\right)^{\sigma_{3}}\left(\begin{array}{cc}
-i & i \\
1 & 1
\end{array}\right)\left(\frac{N q(b)}{2} b\right)^{-\sigma_{3} / 6} .
$$


Thus, after employing a local contour deformation, we derive that the parametrix $U(z)$ has jumps along the curves depicted in Figure 6. Moreover, these jumps are described by the same matrices as in the $S$-RHP, indeed with orientation as in Figure 6

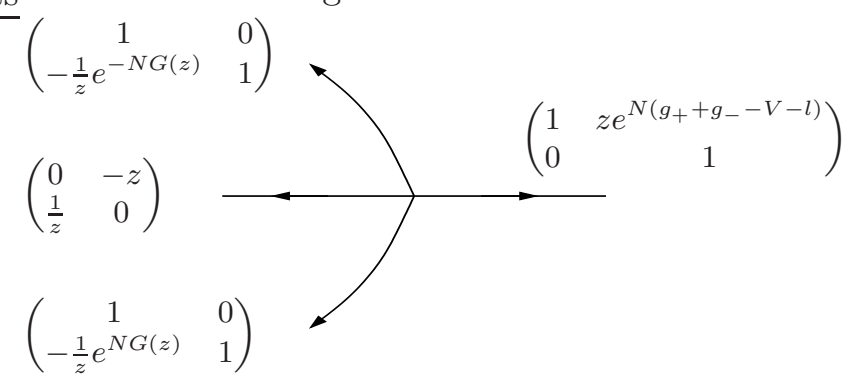

FIgURE 6. Transformation of parametrix jumps to original jumps

$$
\begin{aligned}
& z^{\sigma_{3} / 2} e^{-\frac{2}{3} \zeta^{3 / 2}(z) \sigma_{3}}\left(\begin{array}{cc}
1 & 0 \\
-1 & 1
\end{array}\right) e^{\frac{2}{3} \zeta^{3 / 2}(z) \sigma_{3}} z^{-\sigma_{3} / 2}=\left(\begin{array}{cc}
1 & 0 \\
-\frac{1}{z} e^{-N G(z)} & 1
\end{array}\right), \quad z \in \mathcal{U} \cap \gamma^{+} \\
& z^{\sigma_{3} / 2} e^{-\frac{2}{3} \zeta^{3 / 2}(z) \sigma_{3}}\left(\begin{array}{cc}
0 & -1 \\
1 & 0
\end{array}\right) e^{\frac{2}{3} \zeta^{3 / 2}(z) \sigma_{3}} z^{-\sigma_{3} / 2}=\left(\begin{array}{cc}
0 & -z \\
\frac{1}{z} & 0
\end{array}\right), \quad z \in \mathcal{U} \cap(0, b) \\
& z^{\sigma_{3} / 2} e^{-\frac{2}{3} \zeta^{3 / 2}(z) \sigma_{3}}\left(\begin{array}{ll}
1 & 1 \\
0 & 1
\end{array}\right) e^{\frac{2}{3} \zeta^{3 / 2}(z) \sigma_{3}} z^{-\sigma_{3} / 2}=\left(\begin{array}{cc}
1 & z e^{N\left(g_{+}+g_{-}-V-l\right)} \\
0 & 1
\end{array}\right), \quad z \in \mathcal{U} \cap(b, \infty) \\
& z^{\sigma_{3} / 2} e^{-\frac{2}{3} \zeta^{3 / 2}(z) \sigma_{3}}\left(\begin{array}{cc}
1 & 0 \\
-1 & 1
\end{array}\right) e^{\frac{2}{3} \zeta^{3 / 2}(z) \sigma_{3}} z^{-\sigma_{3} / 2}=\left(\begin{array}{cc}
1 & 0 \\
-\frac{1}{z} e^{N G(z)} & 1
\end{array}\right), \quad z \in \mathcal{U} \cap \gamma^{-} .
\end{aligned}
$$

But this means that the ratio of $S(z)$ with $U(z)$ is locally analytic (here we use the boundedness of the Airy function at the origin), i.e.

$$
S(z)=N_{r}(z) U(z), \quad|z-b|<r<\frac{b}{2} .
$$

The use of the multiplier $B_{r}(z)$ in the definition (3.18) follows from the need of a "matching" between the local model functions $U(z)$ and $M(z)$ : observe that

$$
B_{r}(z)\left(-\frac{i}{2}\right) \zeta^{\sigma_{3} / 4}(z)\left(\begin{array}{cc}
-1 & i \\
1 & i
\end{array}\right)=M(z) z^{\sigma_{3} / 2}
$$

so that with the asymptotics (3.16),

$$
\begin{aligned}
U(z) & =M(z) z^{\sigma_{3} / 2}\left[I+\frac{1}{48 \zeta^{3 / 2}}\left(\begin{array}{cc}
1 & 6 i \\
6 i & -1
\end{array}\right)+\frac{35}{4608 \zeta^{6 / 2}}\left(\begin{array}{cc}
-1 & 12 i \\
-12 i & -1
\end{array}\right)+O\left(\zeta^{-9 / 2}\right)\right] z^{-\sigma_{3} / 2} \\
& =\left[I+\frac{U_{1}(z)}{96 \zeta^{3 / 2}}+\frac{35 U_{2}(z)}{4608 \zeta^{6 / 2}}+O\left(\zeta^{-9 / 2}\right)\right] M(z)
\end{aligned}
$$

as $N \rightarrow \infty$ for any $\alpha>1$ and $0<r_{1} \leq|z-b| \leq r_{2}<\frac{b}{2}$ (so $|\zeta| \rightarrow \infty$ ). Here $U_{k}=\left(U_{k}^{i j}\right)$ are given by

$$
\begin{aligned}
U_{1}^{11}(z)= & \delta^{2}(z)\left(1-\frac{3}{z} \mathcal{D}^{2}(z)-3 z \mathcal{D}^{-2}(z)\right)+\delta^{-2}(z)\left(1+\frac{3}{z} \mathcal{D}^{2}(z)+3 z \mathcal{D}^{-2}(z)\right)=-U_{1}^{22} \\
U_{1}^{12}(z)= & -\frac{i b}{4}\left(\delta^{2}(z)-\delta^{-2}(z)-\frac{3}{z} \mathcal{D}^{2}(z)\left(\delta(z)-\delta^{-1}(z)\right)^{2}\right. \\
& \left.-3 z \mathcal{D}^{-2}(z)\left(\delta(z)+\delta^{-1}(z)\right)^{2}\right)
\end{aligned}
$$




$$
\begin{aligned}
U_{1}^{21}(z)= & -\frac{4 i}{b}\left(\delta^{2}(z)-\delta^{-2}(z)-\frac{3}{z} \mathcal{D}^{2}(z)\left(\delta(z)+\delta^{-1}(z)\right)^{2}\right. \\
& \left.-3 z \mathcal{D}^{-2}(z)\left(\delta(z)-\delta^{-1}(z)\right)^{2}\right)
\end{aligned}
$$

and

$$
\begin{aligned}
& U_{2}^{11}(z)=-1+\delta^{2}(z)\left(\frac{3}{z} \mathcal{D}^{2}(z)-3 z \mathcal{D}^{-2}(z)\right)-\delta^{-2}(z)\left(\frac{3}{z} \mathcal{D}^{2}(z)-3 z \mathcal{D}^{-2}(z)\right) \\
& U_{2}^{22}(z)=-1-\delta^{2}(z)\left(\frac{3}{z} \mathcal{D}^{2}(z)-3 z \mathcal{D}^{-2}(z)\right)+\delta^{-2}(z)\left(\frac{3}{z} \mathcal{D}^{2}(z)-3 z \mathcal{D}^{-2}(z)\right) \\
& U_{2}^{12}(z)=-\frac{i b}{4}\left(\frac{3}{z} \mathcal{D}^{2}(z)\left(\delta(z)-\delta^{-1}(z)\right)^{2}-3 z \mathcal{D}^{-2}(z)\left(\delta(z)+\delta^{-1}(z)\right)^{2}\right) \\
& U_{2}^{21}(z)=-\frac{4 i}{b}\left(\frac{3}{z} \mathcal{D}^{2}(z)\left(\delta(z)+\delta^{-1}(z)\right)^{2}-3 z \mathcal{D}^{-2}(z)\left(\delta(z)-\delta^{-1}(z)\right)^{2}\right) .
\end{aligned}
$$

But as the function $\zeta(z)$ is of order $N^{2 / 3}$ on the latter annulus and $\delta(z), \mathcal{D}(z)$ are bounded, we obtain an asymptotical matching between the model functions from equation (3.20),

$$
U(z)=(I+o(1)) M(z), \quad N \rightarrow \infty, \quad 0 \leq \tau \leq 1-\varepsilon<1, \quad 0<r_{1} \leq|z-b| \leq r_{2}<\frac{b}{2}
$$

The latter relation will be important later on and we also emphasize that the last estimation is uniform with respect to the parameter $0 \leq \tau \leq 1-\varepsilon<1$.

3.6. Construction of a parametrix at $z=0$. Fix a small neighborhood $\mathcal{V}$ of the origin and observe that

$$
\begin{aligned}
G(z)=2 g(z)-V(z)-l & =\int_{z}^{b} \sqrt{\frac{w-b}{w}} q(w) d w=2 \pi i-\int_{0}^{z} \sqrt{\frac{w-b}{w}} q(w) d w \\
& =2 \pi i-2 h_{2}(z) \sqrt{z}, \quad z \in \mathcal{V} \cap \gamma^{+}, \quad 0<\arg z \leq 2 \pi
\end{aligned}
$$

where $h_{2}(z)$ is analytic in $\mathcal{V}$ such that

Similarly

$$
h_{2}(z)=e^{i \frac{\pi}{2}} q(0) \sqrt{b}\left[1+\frac{1}{3}\left(\frac{q^{\prime}(0)}{q(0)}-\frac{1}{2 b}\right) z+O\left(z^{2}\right)\right], \quad z \rightarrow 0 .
$$

$$
G(z)=-2 g(z)+V(z)+l=2 \pi i+2 h_{2}(z) \sqrt{z}, \quad z \in \mathcal{V} \cap \gamma^{-}, \quad 0<\arg z \leq 2 \pi
$$

and both stated local behaviors suggest to use the Bessel functions $I_{1}(\zeta)$ and $K_{1}(\zeta)$ in the construction of an edge point parametrix. Again, we proceed in several steps. First we recall (cf. [4]) that the modified Bessel functions are unique independent solutions to Bessel's equation

$$
z^{2} w^{\prime \prime}+z w^{\prime}-\left(z^{2}+1\right) w=0
$$

satisfying the following asymptotic conditions as $\zeta \rightarrow \infty$ and $-\frac{\pi}{2}<\arg \zeta<\frac{3 \pi}{2}$

$$
I_{1}(\zeta) \sim \frac{e^{\zeta}}{\sqrt{2 \pi \zeta}}\left(1-\frac{3}{8 \zeta}-\frac{15}{64 \zeta^{2}}+O\left(\zeta^{-3}\right)\right)+\frac{e^{-\zeta} e^{i \frac{3 \pi}{2}}}{\sqrt{2 \pi \zeta}}\left(1+\frac{3}{8 \zeta}-\frac{15}{64 \zeta^{2}}+O\left(\zeta^{-3}\right)\right)
$$

as well as for $-\frac{3 \pi}{2}<\arg \zeta<\frac{\pi}{2}$

$$
I_{1}(\zeta) \sim \frac{e^{\zeta}}{\sqrt{2 \pi \zeta}}\left(1-\frac{3}{8 \zeta}-\frac{15}{64 \zeta^{2}}+O\left(\zeta^{-3}\right)\right)+\frac{e^{-\zeta} e^{-i \frac{3 \pi}{2}}}{\sqrt{2 \pi \zeta}}\left(1+\frac{3}{8 \zeta}-\frac{15}{64 \zeta^{2}}+O\left(\zeta^{-3}\right)\right) .
$$


On the other hand

$$
K_{1}(\zeta)=\sqrt{\frac{\pi}{2 \zeta}} e^{-\zeta}\left(1+\frac{3}{8 \zeta}-\frac{15}{64 \zeta^{2}}+O\left(\zeta^{-3}\right)\right), \quad \zeta \rightarrow \infty
$$

which holds in a full neighborhood of infinity. Secondly $I_{1}(\zeta), K_{1}(\zeta)$ satisfy monodromy relations, valid on the entire universal covering of the punctured plane

$$
I_{1}\left(e^{-i \pi} \zeta\right)=e^{-i \pi} I_{1}(\zeta), \quad K_{1}\left(e^{-i \pi} \zeta\right)=e^{i \pi} K_{1}(\zeta)+i \pi I_{1}(\zeta)
$$

and finally the following expansions at the origin are valid

$$
I_{1}(\zeta)=\frac{\zeta}{2}\left(1+\frac{\zeta^{2}}{8}+O\left(\zeta^{4}\right)\right), \quad K_{1}(\zeta)=\frac{1}{\zeta}\left(1+O\left(\zeta^{2} \ln \zeta\right)\right), \quad \zeta \rightarrow 0 .
$$

Remembering the latter properties we introduce on the punctured plane $\zeta \in \mathbb{C} \backslash\{0\}$,

$$
P_{B E}(\zeta)=e^{-i \frac{\pi}{4}}\left(\begin{array}{cc}
I_{1}\left(2 e^{-i \frac{\pi}{2}} \sqrt{\zeta}\right) & -\frac{i}{\pi} K_{1}\left(2 e^{-i \frac{\pi}{2}} \sqrt{\zeta}\right) \\
-2 \pi i \sqrt{\zeta}\left(I_{1}\right)^{\prime}\left(2 e^{-i \frac{\pi}{2}} \sqrt{\zeta}\right) & -2 \sqrt{\zeta}\left(K_{1}\right)^{\prime}\left(2 e^{-i \frac{\pi}{2}} \sqrt{\zeta}\right)
\end{array}\right), \quad 0<\arg \zeta \leq 2 \pi .
$$

From the behavior of $I_{1}(\zeta)$ and $K_{1}(\zeta)$ at infinity, we deduce

$$
\begin{aligned}
P_{B E}(\zeta)= & \zeta^{-\sigma_{3} / 4}(2 \pi)^{-\sigma_{3} / 2} \frac{1}{\sqrt{2}}\left(\begin{array}{cc}
1 & -i \\
-i & 1
\end{array}\right)\left[I+\frac{1}{16 \sqrt{\zeta}}\left(\begin{array}{cc}
-5 i & -2 \\
-2 & 5 i
\end{array}\right)+\frac{3}{64 \zeta}\left(\begin{array}{cc}
-1 & -6 i \\
6 i & -1
\end{array}\right)\right. \\
& \left.+O\left(\zeta^{-3 / 2}\right)\right] e^{-2 i \sqrt{\zeta} \sigma_{3}}\left(\begin{array}{cc}
1 & 0 \\
1 & 1
\end{array}\right), \quad \zeta \rightarrow \infty, \frac{\pi}{3}<\arg \zeta<\frac{7 \pi}{3}
\end{aligned}
$$

and

$$
\begin{aligned}
P_{B E}(\zeta)= & \zeta^{-\sigma_{3} / 4}(2 \pi)^{-\sigma_{3} / 2} \frac{1}{\sqrt{2}}\left(\begin{array}{cc}
1 & -i \\
-i & 1
\end{array}\right)\left[I+\frac{1}{16 \sqrt{\zeta}}\left(\begin{array}{cc}
-5 i & -2 \\
-2 & 5 i
\end{array}\right)+\frac{3}{64 \zeta}\left(\begin{array}{cc}
-1 & -6 i \\
6 i & -1
\end{array}\right)\right. \\
& \left.+O\left(\zeta^{-3 / 2}\right)\right] e^{-2 i \sqrt{\zeta} \sigma_{3}}\left(\begin{array}{cc}
1 & 0 \\
-1 & 1
\end{array}\right), \quad \zeta \rightarrow \infty,-\frac{\pi}{3}<\arg \zeta<\frac{5 \pi}{3} .
\end{aligned}
$$

The "bare parametrix" is given by

$$
P_{B E}^{R H}(\zeta)= \begin{cases}P_{B E}(\zeta)\left(\begin{array}{cc}
1 & 0 \\
1 & 1
\end{array}\right), & \arg \zeta \in\left(0, \frac{\pi}{3}\right), \\
P_{B E}(\zeta), & \arg \zeta \in\left(\frac{\pi}{3}, \frac{5 \pi}{3}\right), \\
P_{B E}(\zeta)\left(\begin{array}{cc}
1 & 0 \\
-1 & 1
\end{array}\right), & \arg \zeta \in\left(\frac{5 \pi}{3}, 2 \pi\right) .\end{cases}
$$

and its analytical properties summarized below

- $P_{B E}^{R H}(\zeta)$ is analytic for $\zeta \in \mathbb{C} \backslash\left\{\arg \zeta=0, \frac{\pi}{3}, \frac{5 \pi}{3}\right\}$

- The following jumps hold, see Figure 7 .

$$
\begin{array}{ll}
\left(P_{B E}^{R H}(\zeta)\right)_{+}=\left(P_{B E}^{R H}(\zeta)\right)_{-}\left(\begin{array}{cc}
1 & 0 \\
-1 & 1
\end{array}\right), & \arg \zeta=\frac{\pi}{3} \\
\left(P_{B E}^{R H}(\zeta)\right)_{+}=\left(P_{B E}^{R H}(\zeta)\right)_{-}\left(\begin{array}{cc}
1 & 0 \\
-1 & 1
\end{array}\right), & \arg \zeta=\frac{5 \pi}{3} .
\end{array}
$$

For the jump on the line $\arg \zeta=0$ we notice that the monodromy relations imply

$$
\begin{aligned}
I_{1}\left(2 e^{-i \frac{\pi}{2}} \sqrt{\zeta}_{+}\right) & =I_{1}\left(2 e^{-i \frac{\pi}{2}} e^{-i \pi} \sqrt{\zeta}_{-}\right)=e^{-i \pi} I_{1}\left(2 e^{-i \frac{\pi}{2}} \sqrt{\zeta}_{-}\right) \\
\left(I_{1}\right)^{\prime}\left(2 e^{-i \frac{\pi}{2}} \sqrt{\zeta_{+}}\right) & =\left(I_{1}\right)^{\prime}\left(2 e^{-i \frac{\pi}{2}} \sqrt{\zeta}_{-}\right)
\end{aligned}
$$




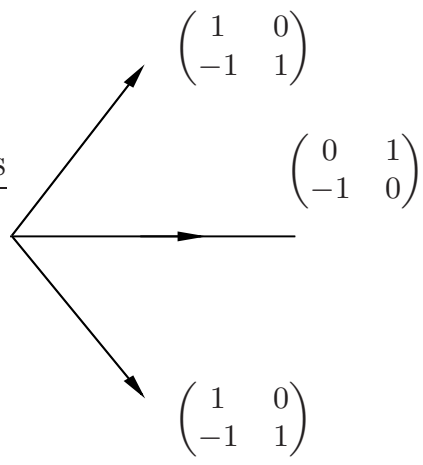

FiguRE 7 . The model RHP near $z=0$ which can be solved explicitly using Bessel functions

and

$$
\begin{aligned}
K_{1}\left(2 e^{-i \frac{\pi}{2}} \sqrt[\zeta_{+}]{ }\right) & =K_{1}\left(2 e^{-i \frac{\pi}{2}} e^{-i \pi} \sqrt{\zeta}_{-}\right)=e^{i \pi} K_{1}\left(2 e^{-i \frac{\pi}{2}} \sqrt{\zeta}\right)+i \pi I_{1}\left(2 e^{-i \frac{\pi}{2}} \sqrt{\zeta}\right) \\
\left(K_{1}\right)^{\prime}\left(2 e^{-i \frac{\pi}{2}} \sqrt{\zeta_{+}}\right) & =\left(K_{1}\right)^{\prime}\left(2 e^{-i \frac{\pi}{2}} \sqrt{\zeta}_{-}\right)-i \pi\left(I_{1}\right)^{\prime}\left(2 e^{-i \frac{\pi}{2}} \sqrt{\zeta_{-}}\right) .
\end{aligned}
$$

Therefore

$$
\left(P_{B E}(\zeta)\right)_{+}=\left(P_{B E}(\zeta)\right)_{-}\left(\begin{array}{cc}
-1 & 1 \\
0 & -1
\end{array}\right)
$$

and hence

$$
\left(P_{B E}^{R H}(\zeta)\right)_{+}=\left(P_{B E}^{R H}(\zeta)\right)_{-}\left(\begin{array}{cc}
0 & 1 \\
-1 & 0
\end{array}\right), \quad \arg \zeta=0
$$

- At the origin

$$
P_{B E}^{R H}(\zeta)=\frac{e^{-i \frac{\pi}{4}}}{2 \sqrt{\zeta}}\left[\left(\begin{array}{cc}
0 & \frac{1}{\pi} \\
0 & 1
\end{array}\right)+O(\zeta \ln \zeta)\right], \quad \zeta \rightarrow 0, \quad \frac{\pi}{3}<\arg \zeta<\frac{5 \pi}{3}
$$

and for the other sector we have to multiply the latter expansion with the correct multipliers from (3.26)

- In order to determine the behavior of $P_{B E}^{R H}(\zeta)$ at infinity, we recall (3.24) and (3.25) as well as

$$
e^{-2 i \sqrt{\zeta} \sigma_{3}}\left(\begin{array}{cc}
1 & 0 \\
\pm 1 & 1
\end{array}\right) e^{2 i \sqrt{\zeta} \sigma_{3}}=\left(\begin{array}{cc}
1 & 0 \\
\pm e^{4 i \sqrt{\zeta}} & 1
\end{array}\right), \quad \frac{\pi}{3}<\arg \zeta<\frac{5 \pi}{3}
$$

However for those $\zeta$, we have $\operatorname{Re}(4 i \sqrt{\zeta})<0$, hence the given product approaches the identity exponentially fast as $\zeta \rightarrow \infty$. Together we have

$$
\begin{aligned}
P_{B E}^{R H}(\zeta)= & \zeta^{-\sigma_{3} / 4}(2 \pi)^{-\sigma_{3} / 2} \frac{1}{\sqrt{2}}\left(\begin{array}{cc}
1 & -i \\
-i & 1
\end{array}\right)\left[I+\frac{1}{16 \sqrt{\zeta}}\left(\begin{array}{cc}
-5 i & -2 \\
-2 & 5 i
\end{array}\right)\right. \\
& \left.+\frac{3}{64 \zeta}\left(\begin{array}{cc}
-1 & -6 i \\
6 i & -1
\end{array}\right)+O\left(\zeta^{-3 / 2}\right)\right] e^{-2 i \sqrt{\zeta} \sigma_{3}}, \quad \zeta \rightarrow \infty
\end{aligned}
$$

valid in a whole neighborhood of infinity.

With the help of the model function $P_{B E}^{R H}(\zeta)$, the local parametrix near $z=0$ is now defined as follows: first define

$$
\zeta(z)=e^{-i \pi}\left(\frac{N}{4}\right)^{2}(-2 g(z)+V(z)+l-2 \pi i)^{2}, \quad|z|<r, \quad 0<\arg \zeta \leq 2 \pi .
$$


which is also a locally conformal change of variables, as

$$
\zeta(z)=\left(\frac{N q(0) \sqrt{b}}{2}\right)^{2} z\left[1+\frac{2}{3}\left(\frac{q^{\prime}(0)}{q(0)}-\frac{1}{2 b}\right) z+O\left(z^{2}\right)\right], \quad|z|<r .
$$

Using the change $\zeta=\zeta(z)$, the left parametrix $V(z)$ near $z=0$ is given by the formula

$$
W(z)=B_{l}(z) P_{B E}^{R H}(\zeta(z)) e^{2 i \zeta^{1 / 2}(z) \sigma_{3}}(-z)^{-\sigma_{3} / 2}, \quad|z|<r
$$

with the matrix multiplier

$$
B_{l}(z)=M(z)(-z)^{\sigma_{3} / 2} \frac{1}{\sqrt{2}}\left(\begin{array}{cc}
1 & i \\
i & 1
\end{array}\right) \zeta^{\sigma_{3} / 4}(z)(2 \pi)^{\sigma_{3} / 2} .
$$

Again $B_{l}(z)$ is analytic in a neighborhood of $z=0$, for $z \in(0, r)$

$$
\begin{aligned}
\left(B_{l}(z)\right)_{+} & =M_{+}(z)(-z)_{+}^{\sigma_{3} / 2} \frac{1}{\sqrt{2}}\left(\begin{array}{cc}
1 & i \\
i & 1
\end{array}\right) \zeta_{+}^{\sigma_{3} / 4}(z)(2 \pi)^{\sigma_{3} / 2} \\
& =M_{-}(z)\left(\begin{array}{cc}
0 & z \\
-\frac{1}{z} & 0
\end{array}\right)(-z)_{-}^{\sigma_{3} / 2} e^{i \pi \sigma_{3}} \frac{1}{\sqrt{2}}\left(\begin{array}{cc}
1 & i \\
i & 1
\end{array}\right) \zeta_{-}^{\sigma_{3} / 4}(z) e^{-i \frac{\pi}{2} \sigma_{3}}(2 \pi)^{\sigma_{3} / 2} \\
& =\left(B_{l}(z)\right)_{-}
\end{aligned}
$$

and with

$$
\delta(z)(\zeta(z))^{-1 / 4}=\left(\frac{z}{\zeta(z)(z-b)}\right)^{1 / 4}=\left(\frac{4 e^{i \pi}}{N^{2} b^{2} q(0)}\right)^{1 / 4}(1+O(z)), \quad z \rightarrow 0
$$

we obtain from a direct computation

$$
B_{l}(0)=\frac{1}{\sqrt{2}}\left(\frac{\sqrt{b}}{2}\right)^{\sigma_{3}}\left(\begin{array}{ll}
1 & i \\
i & 1
\end{array}\right)\left(\frac{N^{2} b^{2} q(0)}{4} e^{-i \pi}\right)^{\sigma_{3} / 4} .
$$

Moreover the latter identity combined with (3.27), allows us to show that

as well as

$$
W(z)=O(1), \quad z \rightarrow 0, \quad \frac{\pi}{3}<\arg z<\frac{5 \pi}{3}
$$

$$
W(z)=O\left(z^{-1}\right), \quad z \rightarrow 0, \quad 0<\arg z<\frac{\pi}{3}, \quad \frac{5 \pi}{3}<\arg z<2 \pi,
$$

which precisely matches the endpoint behavior of $S(z)$ in (3.8) and (3.9). On the other hand, the parametrix $W(z)$ has jumps along the curves depicted in Figure 8 , and we can locally match the latter curves with the jump contour in the S-RHP.

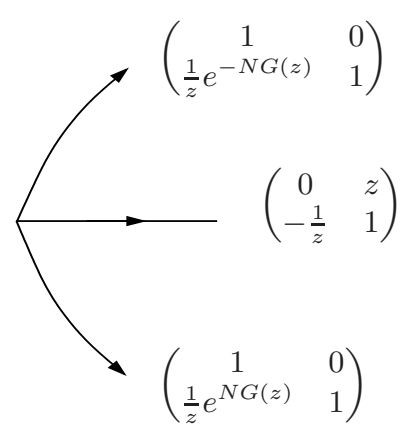

FIGURE 8. Transformation of parametrix jumps to original jumps 
Moreover, the jumps are identical to the ones in the $S$-RHP since

$$
\begin{aligned}
& (-z)^{\sigma_{3} / 2} e^{-2 i \zeta^{1 / 2}(z) \sigma_{3}}\left(\begin{array}{cc}
1 & 0 \\
-1 & 1
\end{array}\right) e^{2 i \zeta^{1 / 2}(z) \sigma_{3}}(-z)^{-\sigma_{3} / 2}=\left(\begin{array}{cc}
1 & 0 \\
\frac{1}{z} e^{-N G(z)} & 1
\end{array}\right), \quad z \in \mathcal{V} \cap \gamma^{+} \\
& (-z)^{\sigma_{3} / 2} e^{-2 i \zeta^{1 / 2}(z) \sigma_{3}}\left(\begin{array}{cc}
0 & 1 \\
-1 & 0
\end{array}\right) e^{2 i \zeta^{1 / 2}(z) \sigma_{3}}(-z)^{-\sigma_{3} / 2}=\left(\begin{array}{cc}
0 & z \\
-\frac{1}{z} & 0
\end{array}\right), \quad z \in \mathcal{V} \cap(0, b) \\
& (-z)^{\sigma_{3} / 2} e^{-2 i \zeta^{1 / 2}(z) \sigma_{3}}\left(\begin{array}{cc}
1 & 0 \\
-1 & 1
\end{array}\right) e^{2 i \zeta^{1 / 2}(z) \sigma_{3}}(-z)^{-\sigma_{3} / 2}=\left(\begin{array}{cc}
1 & 0 \\
\frac{1}{z} e^{N G(z)} & 1
\end{array}\right), \quad z \in \mathcal{V} \cap \gamma^{-}
\end{aligned}
$$

Hence the ratio of $S(z)$ with $W(z)$ is locally analytic, i.e.

$$
S(z)=N_{l}(z) W(z), \quad 0<|z|<r<\frac{b}{2}
$$

The role of the left multiplier $B_{l}(z)$ in (3.30) is the same as in the construction of the parametrix $U(z)$, it provides us with an asymptotic matching relation between the model functions: with

$$
B_{l}(z) \zeta^{-\sigma_{3} / 4}(z)(2 \pi)^{-\sigma_{3} / 2} \frac{1}{\sqrt{2}}\left(\begin{array}{cc}
1 & -i \\
-i & 1
\end{array}\right)=M(z)(-z)^{\sigma_{3} / 2}
$$

we deduce from (3.28)

$$
\begin{aligned}
W(z) & =M(z)(-z)^{\sigma_{3} / 2}\left[I+\frac{1}{16 \sqrt{\zeta}}\left(\begin{array}{cc}
-5 i & -2 \\
-2 & 5 i
\end{array}\right)+\frac{3}{64 \zeta}\left(\begin{array}{cc}
-1 & -6 i \\
6 i & -1
\end{array}\right)+O\left(\zeta^{-3 / 2}\right)\right](-z)^{-\sigma_{3} / 2} \\
& =\left[I+\frac{W_{1}(z)}{32 \sqrt{\zeta}}+\frac{3 W_{2}(z)}{64 \zeta}+O\left(\zeta^{-3 / 2}\right)\right] M(z)
\end{aligned}
$$

as $N \rightarrow \infty$ (hence $|\zeta| \rightarrow \infty$ ), for any $\alpha>1$ and $0<r_{1} \leq|z| \leq r_{2}<\frac{b}{2}$. The coefficients $W_{k}=\left(W_{k}^{i j}\right)$ are given by

$$
\begin{aligned}
W_{1}^{11}(z)= & -i \delta^{2}(z)\left(5-\frac{1}{z} \mathcal{D}^{2}(z)-z \mathcal{D}^{-2}(z)\right)-i \delta^{-2}(z)\left(5+\frac{1}{z} \mathcal{D}^{2}(z)+z \mathcal{D}^{-2}(z)\right) \\
= & -W_{1}^{22}(z), \\
W_{1}^{12}(z)= & \frac{b}{4}\left(-5\left(\delta^{2}(z)-\delta^{-2}(z)\right)+\frac{1}{z} \mathcal{D}^{2}(z)\left(\delta(z)-\delta^{-1}(z)\right)^{2}\right. \\
& \left.+z \mathcal{D}^{-2}(z)\left(\delta(z)+\delta^{-1}(z)\right)^{2}\right), \\
W_{1}^{21}(z)= & \frac{4}{b}\left(-5\left(\delta^{2}(z)-\delta^{-2}(z)\right)+\frac{1}{z} \mathcal{D}^{2}(z)\left(\delta(z)+\delta^{-1}(z)\right)^{2}\right. \\
& \left.+z \mathcal{D}^{-2}(z)\left(\delta(z)-\delta^{-1}(z)\right)^{2}\right),
\end{aligned}
$$


and

$$
\begin{aligned}
& W_{2}^{11}(z)=-1+\frac{3}{2} \delta^{2}(z)\left(\frac{1}{z} \mathcal{D}^{2}(z)-z \mathcal{D}^{-2}(z)\right)-\frac{3}{2} \delta^{-2}(z)\left(\frac{1}{z} \mathcal{D}^{2}(z)-z \mathcal{D}^{-2}(z)\right), \\
& W_{2}^{22}(z)=-1-\frac{3}{2} \delta^{2}(z)\left(\frac{1}{z} \mathcal{D}^{2}(z)-z \mathcal{D}^{-2}(z)\right)+\frac{3}{2} \delta^{-2}(z)\left(\frac{1}{z} \mathcal{D}^{2}(z)-z \mathcal{D}^{-2}(z)\right), \\
& W_{2}^{12}(z)=-\frac{i b}{4}\left(\frac{3}{2 z} \mathcal{D}^{2}(z)\left(\delta(z)-\delta^{-1}(z)\right)^{2}-\frac{3}{2} z \mathcal{D}^{-2}(z)\left(\delta(z)+\delta^{-1}(z)\right)^{2}\right), \\
& W_{2}^{21}(z)=-\frac{4 i}{b}\left(\frac{3}{2 z} \mathcal{D}^{2}(z)\left(\delta(z)+\delta^{-1}(z)\right)^{2}-\frac{3}{2} z \mathcal{D}^{-2}(z)\left(\delta(z)-\delta^{-1}(z)\right)^{2}\right) .
\end{aligned}
$$

Since $\zeta(z)$ is of order $N^{2}$ on the latter annulus and $\delta(z), \mathcal{D}(z)$ are bounded, equation (3.31) implies the following matching relation between $W(z)$ and $M(z)$,

$$
W(z)=(I+o(1)) M(z), \quad N \rightarrow \infty, 0 \leq \tau \leq 1-\varepsilon<1, \quad 0<r_{1} \leq|z| \leq r_{2}<\frac{b}{2} .
$$

We now use the model functions $M(z), U(z)$ and $W(z)$ and employ another transformation.

3.7. Third transformation of the RHP - ratio problem. In this step we put

$$
R(z)=S(z) \begin{cases}(W(z))^{-1}, & |z|<r_{1} \\ (U(z))^{-1}, & |z-b|<r_{2} \\ (M(z))^{-1}, & |z|>r_{1},|z-b|>r_{2}\end{cases}
$$

with $0<r_{2}<\frac{b}{2}$ and $0 \leq r_{1}<\min \left\{\frac{\pi}{2 t}, \frac{b}{2}\right\}$. The reason for choosing the latter radius in this explicit $t$-dependent form arises from the analyticity of the potential $V(z)$, which is holomorphic in the strip $\Delta_{t}$. Moreover the set of its branch points $\Omega_{t}$ is given by

$$
\Omega_{t}=\left\{i \frac{n \pi}{t}: n \in \mathbb{Z} \backslash\{0\}\right\} .
$$

Hence we need to choose a neighborhood of the origin in (3.34) which does not include any of the branch points. With $C_{t, b}$ denoting the clockwise oriented circles shown in Figure 9, the ratiofunction $R(z)$ solves the following RHP

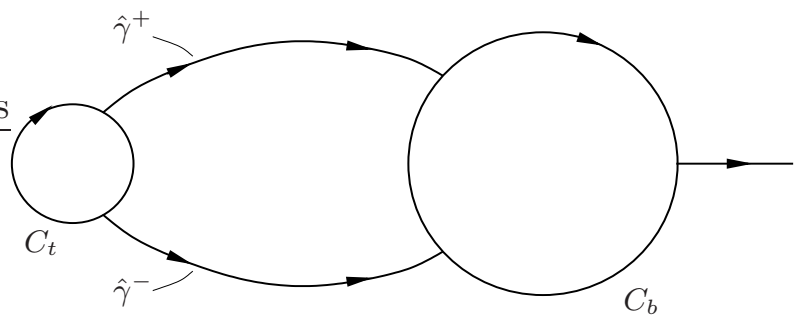

Figure 9. The jump graph for the ratio function $R(z)$

- $R(z)$ is analytic for $z \in \mathbb{C} \backslash\left\{C_{t, b} \cup \hat{\Gamma} \cup\left(b+r_{2}, \infty\right)\right\}$ with $\hat{\Gamma}=\hat{\gamma}_{+} \cup \hat{\gamma}_{-}$

- The jumps are as follows: on the infinite branch $\left(b+r_{2}, \infty\right)$,

$$
R_{+}(z)=R_{-}(z) M(z)\left(\begin{array}{cc}
1 & z e^{N\left(g_{+}+g_{-}-V-l\right)} \\
0 & 1
\end{array}\right)(M(z))^{-1},
$$


on the upper lens boundary $\hat{\gamma}_{+}$, resp. lower lens boundary $\hat{\gamma}_{-}$,

$$
\begin{aligned}
& R_{+}(z)=R_{-}(z) M(z)\left(\begin{array}{cc}
1 & 0 \\
\frac{1}{z} e^{-N G(z)} & 1
\end{array}\right)(M(z))^{-1}, \quad z \in \hat{\gamma}_{+} \\
& R_{+}(z)=R_{-}(z) M(z)\left(\begin{array}{cc}
1 & 0 \\
\frac{1}{z} e^{N G(z)} & 1
\end{array}\right)(M(z))^{-1}, \quad z \in \hat{\gamma}_{-}
\end{aligned}
$$

and on the clockwise oriented circles $C_{t, b}$,

$$
R_{+}(z)=R_{-}(z) \begin{cases}W(z)(M(z))^{-1}, & |z|=r_{1}, \\ U(z)(M(z))^{-1}, & |z-b|=r_{2} .\end{cases}
$$

- As $z \rightarrow \infty$, the function is normalized as $R(z) \rightarrow I$.

We note that $R(z)$ has no jumps inside of $C_{t}$ and $C_{b}$ and across the line segment in between. Also, $R(z)$ is bounded at $z=0$ and $z=b$, which follows from (3.20) and (3.31). To move ahead, we recall the previously stated behavior of the jump matrices as $N \rightarrow \infty$ and note that these estimations are valid for any $\alpha>1$ such that $0 \leq \tau \leq 1-\varepsilon<1$. In fact, on the half ray $\left(b+r_{2}, \infty\right)$, the jumps approach the identity matrix. Through (3.21), the same is also true on the circle $C_{b}$, more precisely with $G_{R}$ denoting the jump matrix in the latter ratio-RHP

$$
\left\|G_{R}-I\right\|_{L^{2} \cap L^{\infty}\left(C_{b}\right)} \leq \frac{\hat{c}}{N}, \quad N \rightarrow \infty, \quad 0 \leq \tau \leq 1-\varepsilon<1
$$

with a constant $\hat{c}>0$ whose value is not important. For the lens boundaries we use the local identities

$$
G(z)=2 \pi i \mp 2 h_{2}(z) \sqrt{z}, \quad z \in \mathcal{V} \cap \gamma^{ \pm}
$$

They imply

$$
\sup _{z \in \hat{\gamma}^{+} \cup \hat{\gamma}^{-}}\left|G_{R}(z)-I\right|=\sup _{z \in\left(\hat{\gamma}^{+} \cup \hat{\gamma}^{-}\right) \cap C_{t}}\left|G_{R}(z)-I\right|=O\left(t e^{-c N t^{-1 / 2}}\right), \quad N \rightarrow \infty, \quad 0 \leq \tau \leq 1-\varepsilon<1
$$

and since

$$
\frac{N}{\sqrt{t}}=\sqrt{N \alpha} \rightarrow \infty \quad \text { as } N \rightarrow \infty \quad \text { for any } \alpha>1: \quad 0 \leq \tau \leq 1-\varepsilon<1,
$$

we see that the contributions arising from the lenses decay exponentially fast. In order to estimate $G_{R}$ on the circle $C_{t}$, we use (3.32)

$$
\sup _{z \in C_{t}}\left|G_{R}(z)-I-\frac{W_{1}(z)}{32 \sqrt{\zeta(z)}}\right|=O\left(\frac{t}{N^{2}}\right)=O\left(\tau N^{-1}\right), \quad N \rightarrow \infty, \quad 0 \leq \tau \leq 1-\varepsilon<1 .
$$

This estimation holds since (3.32) extends to a full asymptotic series of the form

$$
G_{R}(z)-I=\sum_{k=1}^{\infty} \tilde{W}_{k}(z) N^{-k},
$$

valid as long as $N^{2}|z| \rightarrow \infty$. However for $z \in C_{t}$

$$
\frac{1}{N^{2}|z|}=O\left(\tau N^{-1}\right) \quad \text { as } N \rightarrow \infty \quad \text { for any } \alpha>1: 0 \leq \tau \leq 1-\varepsilon<1,
$$

so (3.38) holds in particular for $z \in C_{t}$ and since $\tilde{W}_{k}(z)$ has a pole of order at most $\left\lfloor\frac{k+1}{2}\right\rfloor$ at the origin, we obtain (3.37). From the local expansions

$$
\mathcal{D}^{2}(z)=-z(1+O(\sqrt{z})), \quad \delta^{2}(z)=-i \sqrt{b} \sqrt{z}(1+O(z)), \quad z \rightarrow 0, \quad z \in \mathbb{C} \backslash[0, b]
$$


we now evaluate the residue of $\tilde{W}_{1}(z)$ at the origin

$$
B=\operatorname{res}_{z=0} \tilde{W}_{1}(z)=\operatorname{res}_{z=0}\left(\frac{N W_{1}(z)}{32 \sqrt{\zeta(z)}}\right)=\frac{3}{16 q(0)}\left(\frac{b}{4}\right)^{\sigma_{3} / 2}\left(\begin{array}{cc}
1 & e^{i \frac{\pi}{2}} \\
e^{i \frac{\pi}{2}} & -1
\end{array}\right)\left(\frac{b}{4}\right)^{-\sigma_{3} / 2} .
$$

Hence we can rewrite (3.37) as

$$
\sup _{z \in C_{t}}\left|G_{R}(z)-I-\frac{B}{N z}\right|=O\left(N^{-1}\right), \quad N \rightarrow \infty, \quad 0 \leq \tau \leq 1-\varepsilon<1,
$$

however

$$
\sup _{z \in C_{t}}\left|\frac{B}{N z}\right|=O(\tau), \quad N \rightarrow \infty, \quad 0 \leq \tau \leq 1-\varepsilon<1,
$$

so $G_{R}(z)-I$ is not uniformly close to zero on $C_{t}$ as $N \rightarrow \infty$ for all $0 \leq \tau \leq 1-\varepsilon<1$. To overcome this difficulty we employ our final transformation.

3.8. Fourth and final transformation of the RHP. Since $\operatorname{det} B=\operatorname{trace} B=0$, we see that the matrix function $I+\frac{B}{N z}$ is unimodular for any $z \in \mathbb{C} \backslash\{0\}$, in fact

$$
\left(I+\frac{B}{N z}\right)^{-1}=I-\frac{B}{N z}, \quad z \in \mathbb{C} \backslash\{0\} .
$$

We introduce

$$
Q(z)= \begin{cases}R(z), & |z| \leq r_{1} \\ R(z)\left(I+\frac{B}{N z}\right)^{-1}, & |z|>r_{1}\end{cases}
$$

and are lead to the following RHP

- $Q(z)$ is analytic for $z \in \mathbb{C} \backslash\left\{C_{t, b} \cup \hat{\Gamma} \cup\left(b+r_{2}, \infty\right)\right\}$

- With $G_{Q}$ denoting the jump matrix in the $Q$-RHP we have

$$
\begin{aligned}
& G_{Q}(z)=G_{R}(z)\left(I+\frac{B}{N z}\right)^{-1}, \quad z \in C_{t} \\
& G_{Q}(z)=\left(I+\frac{B}{N z}\right) G_{R}(z)\left(I+\frac{B}{N z}\right)^{-1}, \quad z \in C_{b} \cup \hat{\Gamma} \cup\left(b+r_{2}, \infty\right)
\end{aligned}
$$

- As $z \rightarrow \infty$, we have $Q(z) \rightarrow I$

In the stated problem all jump matrices approach the identity matrix as $N \rightarrow \infty$ for any $\alpha>1$ such that $0 \leq \tau \leq 1-\varepsilon<1$. More precisely with $\Sigma_{Q}$ denoting the underlying contour

$$
\left\|G_{Q}-I\right\|_{L^{2} \cap L^{\infty}\left(\Sigma_{Q}\right)} \leq \frac{c}{N}, \quad N \rightarrow \infty, \quad 0 \leq \tau \leq 1-\varepsilon<1
$$

with a constant $c>0$ whose value is not important. This estimation allows us to solve the $Q$-RHP iteratively.

3.9. Solution of the RHP for $Q(z)$ via iteration. The final RHP for the function $Q(z)$ reads as

- $Q(z)$ is analytic for $z \in \mathbb{C} \backslash \Sigma_{Q}$.

- The boundary values on the contour shown in Figure 9 are related via the identity

$$
Q_{+}(z)=Q_{-}(z) G_{Q}(z), \quad z \in \Sigma_{Q} .
$$

- The normalization $Q(z)=I+O\left(z^{-1}\right)$ is valid as $z \rightarrow \infty$ 
and it is equivalent to the singular integral equation

$$
Q_{-}(z)=I+\frac{1}{2 \pi i} \int_{\Sigma_{Q}} Q_{-}(w)\left(G_{Q}(w)-I\right) \frac{d w}{w-z_{-}} .
$$

Through (3.41), we obtain [18, 10] that equation (3.42) can be solved iteratively in $L^{2}\left(\Sigma_{Q}\right)$ for sufficiently large $N$ and $\alpha>1: 0 \leq \tau \leq 1-\varepsilon<1$. Also, the unique solution satisfies

$$
\left\|Q_{-}-I\right\|_{L^{2}\left(\Sigma_{Q}\right)} \leq \frac{c}{N}, \quad N \rightarrow \infty \quad \text { for any } \alpha>1: 0 \leq \tau \leq 1-\varepsilon<1 .
$$

We are now ready to determine the large $N$ asymptotics of the normalizing constants $h_{N, t}$. To this end notice that for $z \in \mathbb{C} \backslash \Sigma_{Q}$

$$
Q(z)=I+\frac{i}{2 \pi z} \int_{\Sigma_{Q}} Q_{-}(w)\left(G_{Q}(w)-I\right) d w+O\left(z^{-2}\right), \quad z \rightarrow \infty
$$

and also as $N \rightarrow \infty$ for any $\alpha>1: 0 \leq \tau \leq 1-\varepsilon<1$ (from (3.41) and (3.43) as well as the previous discussion about exponentially small contributions)

$$
\int_{\Sigma_{Q}} Q_{-}(w)\left(G_{Q}(w)-I\right) d w=\int_{C_{t}}\left(G_{Q}(w)-I\right) d w+\int_{C_{b}}\left(G_{Q}(w)-I\right) d w+O\left(N^{-2}\right) .
$$

\section{Asymptotics of $h_{N, t}$ - Proof of theorem 1.3}

We go back to (3.1)

$$
h_{N, t}=-2 \pi i\left(Y_{1}^{(N)}\right)_{12}
$$

and recall that

$$
Y_{1}^{(N)}=\lim _{z \rightarrow \infty}\left(z\left(Y^{(N)}(z) z^{-N \sigma_{3}}-I\right)\right) .
$$

Now recall the sequence of transformations

$$
Y(z) \equiv Y^{(N)}(z) \mapsto T(z) \mapsto S(z) \mapsto R(z) \mapsto Q(z)
$$

and combine it with the expansion

$$
e^{N\left(g(z)-\frac{l}{2}\right) \sigma_{3}} z^{-N \sigma_{3}}=e^{-\frac{N l}{2} \sigma_{3}}\left(I-\frac{N \sigma_{3}}{z} \int_{0}^{b} w \psi(w) d w+O\left(z^{-2}\right)\right), \quad z \rightarrow \infty .
$$

This gives us for (4.1)

$$
Y_{1}^{(N)}=\lim _{z \rightarrow \infty}\left(z\left(e^{\frac{N l}{2} \sigma_{3}} Q(z)\left(I+\frac{B}{N z}\right) M(z) e^{N\left(g(z)-\frac{l}{2}\right) \sigma_{3}} z^{-N \sigma_{3}}-I\right)\right)
$$

and hence (compare (3.44))

$$
\begin{aligned}
e^{-\frac{N l}{2} \sigma_{3}} Y_{1}^{(N)} e^{\frac{N l}{2} \sigma_{3}}= & -N \sigma_{3} \int_{0}^{b} w \psi(w) d w+\frac{b}{4}\left(\begin{array}{cc}
-1 & \frac{i b}{4} \\
\frac{4}{i b} & 1
\end{array}\right)+\frac{B}{N} \\
& +\frac{i}{2 \pi} \int_{\Sigma_{Q}} Q_{-}(w)\left(G_{Q}(w)-I\right) d w .
\end{aligned}
$$


In view of (3.45), we will now compute the contribution from the circle $C_{b}$. First from (3.21) as $N \rightarrow \infty$ for any $\alpha>1: 0 \leq \tau \leq 1-\varepsilon<1$

$$
\int_{C_{b}} Q_{-}(w)\left(G_{Q}(w)-I\right) d w=\frac{1}{96} \int_{C_{b}}\left(\begin{array}{ll}
U_{11}(w) & U_{12}(w) \\
U_{21}(w) & U_{22}(w)
\end{array}\right) \frac{d w}{\zeta^{3 / 2}(w)}+O\left(N^{-2}\right) .
$$

Now use the local expansions

$$
\begin{aligned}
& U_{1}^{11}(z)=-\frac{5 \sqrt{b}}{\sqrt{z-b}}\left(1+\frac{3}{2 b}(z-b)+O\left((z-b)^{2}\right)\right), \\
& U_{1}^{12}(z)=\frac{5 i b \sqrt{b}}{4 \sqrt{z-b}}\left(1+\frac{91}{10 b}(z-b)+O\left((z-b)^{2}\right)\right), \\
& U_{1}^{21}(z)=\frac{20 i}{\sqrt{b} \sqrt{z-b}}\left(1-\frac{1}{2 b}(z-b)+O\left((z-b)^{2}\right)\right),
\end{aligned}
$$

valid as $z \rightarrow b$, and with (3.17) compute the relevant line integral via residue theorem. We obtain, as $N \rightarrow \infty$,

$$
\begin{aligned}
\int_{C_{b}} Q_{-}(w)\left(G_{Q}(w)-I\right) d w= & -\frac{2 \pi i}{96} \frac{b}{N q(b)}\left(\begin{array}{cc}
6\left(\frac{q^{\prime}(b)}{q(b)}-\frac{3}{b}\right) & \frac{i b}{2}\left[-3 \frac{q^{\prime}(b)}{q(b)}+\frac{47}{b}\right] \\
\frac{8 i}{b}\left[-3 \frac{q^{\prime}(b)}{q(b)}-\frac{1}{b}\right] & -6\left(\frac{q^{\prime}(b)}{q(b)}-\frac{3}{b}\right)
\end{array}\right) \\
& +O\left(N^{-2}\right)
\end{aligned}
$$

which is uniform with respect to the parameter $0 \leq \tau \leq 1-\varepsilon<1$. For the remaining line integral along the circle boundary $C_{t}$ recall (3.32) and (3.39) and deduce, as $N \rightarrow \infty$

$$
\int_{C_{t}} Q_{-}(w)\left(G_{Q}(w)-I\right) d w=\int_{C_{t}}\left(G_{R}(w)-I-\frac{B}{N w}\right)\left(I-\frac{B}{N w}\right) d w+O\left(N^{-2}\right)
$$

which is again uniform with respect to the parameter $0 \leq \tau \leq 1-\varepsilon<1$. Now from (3.32), as $z \rightarrow 0$,

$$
\begin{aligned}
& W_{1}^{11}(z)=\frac{3 \sqrt{b}}{\sqrt{z}}\left(1-\frac{3 z}{2 b}+O\left(z^{2}\right)\right), \quad W_{1}^{12}(z)=\frac{b}{4} \frac{3 i \sqrt{b}}{\sqrt{z}}\left(1+\frac{35 z}{6 b}+O\left(z^{2}\right)\right) \\
& W_{1}^{21}(z)=\frac{4}{b} \frac{3 i \sqrt{b}}{\sqrt{z}}\left(1+\frac{z}{2 b}+O\left(z^{2}\right)\right),
\end{aligned}
$$

hence

$$
\begin{aligned}
\frac{W_{1}(z)}{32 \sqrt{\zeta(z)}}-\frac{B}{N z}= & -\frac{1}{3 N}\left(\frac{q^{\prime}(0)}{q(0)}-\frac{1}{2 b}\right) B+\frac{1}{2 N b} \frac{3}{16 q(0)}\left(\frac{b}{4}\right)^{\sigma_{3} / 2}\left(\begin{array}{cc}
-3 & \frac{35 i}{3} \\
i & 3
\end{array}\right)\left(\frac{b}{4}\right)^{-\sigma_{3} / 2} \\
& +O\left(z N^{-1}\right), \quad z \rightarrow 0 .
\end{aligned}
$$

Back to (4.2), as $N \rightarrow \infty$ therefore

$$
\int_{C_{t}} Q_{-}(w)\left(G_{Q}(w)-I\right) d w=O\left(N^{-2}\right)
$$


which is uniform with respect to the parameter $0 \leq \tau \leq 1-\varepsilon<1$. At this point we summarize our computations

$$
\begin{aligned}
e^{-\frac{N l}{2} \sigma_{3}} Y_{1}^{(N)} e^{\frac{N l}{2} \sigma_{3}}= & -N \sigma_{3} \int_{0}^{b} w \psi(w) d w+\frac{b}{4}\left(\begin{array}{cc}
-1 & \frac{i b}{4} \\
\frac{4}{i b} & 1
\end{array}\right)+\frac{B}{N} \\
& +\frac{1}{96} \frac{b}{N q(b)}\left(\begin{array}{cc}
6\left(\frac{q^{\prime}(b)}{q(b)}-\frac{3}{b}\right) & \frac{i b}{2}\left[-3 \frac{q^{\prime}(b)}{q(b)}+\frac{47}{b}\right] \\
\frac{8 i}{b}\left[-3 \frac{q^{\prime}(b)}{q(b)}-\frac{1}{b}\right] & -6\left(\frac{q^{\prime}(b)}{q(b)}-\frac{3}{b}\right)
\end{array}\right)+O\left(N^{-2}\right),
\end{aligned}
$$

which implies, as $N \rightarrow \infty$,

$$
\left(Y_{1}^{(N)}\right)_{12}=i e^{N l}\left(\frac{b}{4}\right)^{2}\left[1+\frac{v}{N}+O\left(N^{-2}\right)\right], \quad 0 \leq \tau \leq 1-\varepsilon<1,
$$

with (compare (1.29) $)$

$$
v=\frac{3}{4 b q(0)}-\frac{q^{\prime}(b)}{4 q^{2}(b)}+\frac{47}{12 b q(b)} .
$$

All we need to do now is recall (3.1), the connection formula $h_{N}=2 t^{2 N+2} h_{N, t}$ and combine it with Stirling's approximation

$$
N !=\left(\frac{N}{e}\right)^{N} \sqrt{2 \pi N}\left(1+\frac{1}{12 N}+O\left(N^{-2}\right)\right), \quad N \rightarrow \infty .
$$

This gives, as $N \rightarrow \infty$,

$$
\frac{h_{N}}{(N !)^{2}}=\frac{N}{8} \tau^{2 N+2} b^{2} \exp \left[N(l+2)+\frac{v}{N}-\frac{1}{6 N}+\varepsilon_{N}(\tau)\right]
$$

which is uniform with respect to the parameter $0 \leq \tau \leq 1-\varepsilon<1$, thus proving Theorem 1.3 ,

Remark: At this point it is useful to compare the latter expansion to the estimation (1.23) derived in [8]. We obtain from the connection $h_{N}^{o}=(\alpha-1)^{2 N+1} h_{N}$ and (4.3), that, as $N \rightarrow \infty$,

$$
\ln \left[\frac{h_{N}^{o}}{(N !)^{2}}\right]=(2 N+1) \ln (1-\tau)+N(l+2)+\ln \left(\frac{t}{8}\right)+2 \ln b+\frac{v}{N}-\frac{1}{6 N}+\varepsilon_{N}(\tau)
$$

uniformly with respect to $0 \leq \tau \leq 1-\varepsilon<1$. Also, as a consequence of the Riemann-Hilbert analysis presented in the last subsections, the estimation

$$
\left|\varepsilon_{N}\right| \leq \frac{c}{(N+1)^{2}}, \quad c>0
$$

on the error term $\varepsilon_{N}(\tau)$, can in fact be extended to a full asymptotic series in reciprocal integer powers of $N$ which is also uniform with respect to the parameter $0 \leq \tau \leq 1-\varepsilon<1$. Now choose $\alpha$ from any compact subset of the set (1.16) and let $N \rightarrow \infty$, i.e. $t \rightarrow \infty$. In this limit, Proposition 2.2 implies with (2.5),

$$
b=\frac{4}{1-\tau}\left(1-\frac{1}{2 N}+\frac{\zeta(3 / 2)}{8 \sqrt{\pi(r-1)} N^{3 / 2}}+O\left(N^{-5 / 2}\right)\right), \quad N \rightarrow \infty, \quad r=\frac{\alpha+1}{\alpha-1}
$$

which extends to a full asymptotic series in reciprocal half-integer powers of $N$, the error terms being uniform on any compact subset of the set (1.16). Also via (2.21) and (2.20), as $N \rightarrow \infty$,

$$
l=4(1-\ln 2)-\frac{3 b}{2}(1-\tau)+2 \ln b-\frac{2}{N}(1-\ln 2)-\frac{\ln (2 b N \tau)}{N}+O\left(N^{-5 / 2}\right)
$$


and which can also be extended to a full asymptotic series in reciprocal half-integer powers of $N$. Combining the last two expansions,

$$
l=-2-4 \ln 2+2 \ln \left(\frac{4}{1-\tau}\right)-\frac{\ln (2 N)}{N}+\frac{\ln (\alpha-1)}{N}-\frac{\zeta(3 / 2)}{2 \sqrt{\pi(r-1)} N^{3 / 2}}+\frac{1}{4 N^{2}}+O\left(N^{-5 / 2}\right) .
$$

and since from (2.14), as $N \rightarrow \infty$

$$
v=\frac{7}{6}+O\left(N^{-1 / 2}\right)
$$

we can go back to (4.4) and derive

$$
\ln \left[\frac{h_{N}^{o}}{(N !)^{2}}\right]=-\frac{\zeta(3 / 2)}{2 \sqrt{\pi(r-1)} N^{1 / 2}}+\frac{1}{4 N}+O\left(N^{-3 / 2}\right), \quad N \rightarrow \infty
$$

which is uniform on any compact subset of the set (1.16). The last estimation agrees with (1.23) and as we have seen, extends to a full asymptotic series in reciprocal half-integer powers of $N$.

As a first step in the computation of the $N$ independent leading term $C$ in the large $N$ expansion (1.15) of $Z_{N}$, we use the Toda equation.

\section{Toda equation and the Structure of the Constant factor}

We use the Toda equation as written in (1.13),

$$
\left(\ln \tau_{N}\right)^{\prime \prime}=\frac{h_{N}}{h_{N-1}}=\frac{h_{N}^{o}}{(\alpha-1)^{2} h_{N-1}^{o}}, \quad\left({ }^{\prime}\right)=\frac{d}{d \alpha} .
$$

From (4.5) and our discussion thereafter, as $N \rightarrow \infty$,

$$
\ln \left[\frac{h_{N}^{o}}{(N !)^{2}}\right]=-\frac{\zeta(3 / 2)}{2 \sqrt{\pi(r-1)} N^{1 / 2}}+\frac{1}{4 N}+\frac{c_{1}(\alpha)}{N^{3 / 2}}+\frac{c_{2}(\alpha)}{N^{2}}+O\left(N^{-5 / 2}\right)
$$

with some constants $c_{i}(\alpha)$ whose precise form is not important for us. Hence

$$
\ln \left[\frac{h_{N}^{o}}{N^{2} h_{N-1}^{o}}\right]=\frac{\zeta(3 / 2)}{4 \sqrt{\pi(r-1)} N^{3 / 2}}-\frac{1}{4 N^{2}}+O\left(N^{-5 / 2}\right)
$$

and after exponentiating the latter expansion, as $N \rightarrow \infty$

$$
\begin{aligned}
\left(\ln \tau_{N}\right)^{\prime \prime} & =\frac{N^{2}}{(\alpha-1)^{2}}\left(1+\frac{\zeta(3 / 2)}{4 \sqrt{\pi(r-1)} N^{3 / 2}}-\frac{1}{4 N^{2}}+O\left(N^{-5 / 2}\right)\right) \\
& =-N^{2}(\ln (\alpha-1))^{\prime \prime}-\sqrt{N} \frac{\zeta(3 / 2)}{\sqrt{2 \pi}}(\sqrt{\alpha-1})^{\prime \prime}+\frac{1}{4}(\ln (\alpha-1))^{\prime \prime}+O\left(N^{-1 / 2}\right),
\end{aligned}
$$

where the error term is uniform on any compact subset of the set (1.16). Back to (1.14), we have therefore shown that

$$
\left(\ln Z_{N}\right)^{\prime \prime}=N^{2}\left(\ln \left(\frac{\alpha+1}{2}\right)\right)^{\prime \prime}-\sqrt{N} \frac{\zeta(3 / 2)}{\sqrt{2 \pi}}(\sqrt{\alpha-1})^{\prime \prime}+\frac{1}{4}(\ln (\alpha-1))^{\prime \prime}+O\left(N^{-1 / 2}\right) .
$$

On the other hand from (1.14) combined with (4.5),

$$
\ln Z_{N}=\ln C+N^{2} \ln \left(\frac{\alpha+1}{2}\right)-\sqrt{N} \frac{\zeta(3 / 2)}{\sqrt{2 \pi}} \sqrt{\alpha-1}+\frac{1}{4} \ln N+O\left(N^{-1 / 2}\right),
$$

where $C>0$ depends in general on $\alpha$, but not on $N$. Thus, comparing the latter with (5.1), we conclude

$$
\ln C=\frac{1}{4}(\ln (\alpha-1))^{\prime \prime}+O\left(N^{-1 / 2}\right) .
$$


Integrating this expansion, we get

$$
\ln C=\frac{1}{4} \ln (\alpha-1)+d(N) \alpha+c(N)+O\left(N^{-1 / 2}\right)
$$

with some numbers $d(N)$ and $c(N)$ which are independent of $\alpha$. Now choose any distinct $\alpha_{1}, \alpha_{2}$ from (1.16) and derive

$$
\ln C\left(\alpha_{1}\right)-\ln C\left(\alpha_{2}\right)=\frac{1}{4} \ln \left(\alpha_{1}-1\right)-\frac{1}{4} \ln \left(\alpha_{2}-1\right)+d_{1}(N)\left(\alpha_{1}-\alpha_{2}\right)+O\left(N^{-1 / 2}\right),
$$

which shows that the limit

exists and therefore also the limit

$$
\lim _{N \rightarrow \infty} d(N)=d
$$

$$
\lim _{N \rightarrow \infty} c(N)=c .
$$

Taking the limit $N \rightarrow \infty$ in (5.2), we obtain

$$
\ln C=\frac{1}{4} \ln (\alpha-1)+d \alpha+c
$$

and summarize (see (1.17))

Proposition 5.1. The constant factor $C$ in asymptotic formula (1.15) has the form

$$
C=(\alpha-1)^{1 / 4} e^{d \alpha+c} .
$$

In light of the last proposition we now have to compute the remaining two universal constants $c$ and $d$. This will be done by studying two regimes of the double scaling parameter $t=\frac{N}{\alpha}$. First, we are interested in the behavior of the partition function $Z_{N}$ as $N \rightarrow \infty$ and $t$ remains bounded.

\section{The DOUBle SCALING LIMIT OF THE PARTITION FUNCTION}

We start with the observation that

$$
\lim _{\substack{\alpha \rightarrow \infty \\ N \leq N_{0}}} w_{t}(x)=x e^{-N x}
$$

which in particular implies

$$
w_{t}(x) \sim x e^{-N x} \equiv w_{0}(x), \quad t \rightarrow 0 .
$$

The limiting orthogonal polynomials are the normalized (and rescaled) Laguerre polynomials (cf. [4])

for which

$$
p_{n, 0}(x)=\lim _{t \rightarrow 0} p_{n, t}(x)=\frac{(-1)^{n} n !}{N^{n}} L_{n}^{(1)}(N x)
$$

$$
h_{n, t} \sim \int_{0}^{\infty}\left(p_{n, 0}(x)\right)^{2} w_{0}(x) d x=\frac{(n !)^{2}}{N^{2 n+2}} \int_{0}^{\infty}\left(L_{n}^{(1)}(x)\right)^{2} x e^{-x} d x=\frac{(n !)^{2}(n+1)}{N^{2 n+2}} \equiv h_{n, 0}, \quad t \rightarrow 0 .
$$

Let us introduce the abbreviation

$$
\sigma_{N, t}=N^{N(N+1)} \prod_{k=0}^{N-1} \frac{h_{k, t}}{(k !)^{2}}
$$

which satisfies

$$
\lim _{\substack{\alpha \rightarrow \infty \\ N \leq N_{0}}} \sigma_{N, t}=N^{N(N+1)} \prod_{k=0}^{N-1} \frac{h_{k, 0}}{(k !)^{2}}=N !
$$


and which relates to the partition function $Z_{N}$ via the identity

$$
Z_{N}=\left(\frac{\alpha^{2}-1}{2 \alpha}\right)^{N^{2}}\left(\frac{2}{\alpha}\right)^{N} \sigma_{N, t}
$$

We will now evaluate (6.1) by using (4.3), in other words

$$
\begin{aligned}
\sigma_{N, t} & =N^{N(N+1)} h_{0, t} \prod_{k=1}^{N-1} \frac{h_{k, t}}{(k !)^{2}}=N^{2} h_{0, t}\left(\frac{\alpha}{2}\right)^{N-1} \alpha^{N^{2}-1} \prod_{k=1}^{N-1} \frac{h_{k}}{(k !)^{2}} \\
& =N^{2} h_{0, t}(N-1) ! \exp \left[\sum_{k=1}^{N-1}\left(2 \ln \left(\frac{b}{4}\right)+k(l+2)+\frac{v-\frac{7}{6}}{k}+\frac{1}{k}+\varepsilon_{k}(\tau)\right)\right] \\
& =\hat{C}_{0} N^{2} h_{0, t} N ! \exp \left[\sum_{k=1}^{N-1}\left(2 \ln \left(\frac{b}{4}\right)+k(l+2)+\frac{v-\frac{7}{6}}{k}\right)\right]\left(1+O\left(N^{-1}\right)\right),
\end{aligned}
$$

valid as $N \rightarrow \infty$, where the error term is uniform with respect to the parameter $0 \leq \tau \leq 1-\varepsilon<1$ and with a universal, i.e. $N$ and $\tau$ independent constant $\hat{C}_{0}>0$. Now use (1.8) and derive

$$
h_{0, t}=\int_{0}^{\infty}\left(p_{0, t}(x)\right)^{2} w_{t}(x) d x=\hat{c}_{0} \int_{0}^{\infty} w_{t}(x) d x=\frac{\hat{c}_{0}}{N^{2}\left(1-\tau^{2}\right)}
$$

with another universal constant $\hat{c}_{0}>0$. Back to the previous expansion for $\sigma_{N, t}$, as $N \rightarrow \infty$

$$
\sigma_{N, t}=\frac{C_{0} N !}{1-\tau^{2}} \exp \left[\sum_{k=1}^{N-1}\left(2 \ln \left(\frac{b}{4}\right)+k(l+2)+\frac{v-\frac{7}{6}}{k}\right)\right]\left(1+O\left(N^{-1}\right)\right)
$$

which is uniform with respect to the parameter $0 \leq \tau \leq 1-\varepsilon<1$. In order to determine the constant $C_{0}$, we will now evaluate the sums in (6.4) in the double scaling limit $N, \alpha \rightarrow \infty$ with $0 \leq t \leq t_{0}$ and then compare the result with (6.2).

For the sums, use Euler's summation formula

$$
\sum_{k=1}^{N-1} g(k)=\int_{1}^{N-1} g(x) d x+\int_{1}^{N-1} P_{1}(x) g^{\prime}(x) d x+\frac{1}{2}(g(N-1)+g(1)),
$$

which holds for a differentiable function $g: \mathbb{R} \rightarrow \mathbb{R}$ with the Bernoulli polynomial $P_{1}(x)=x-$ $\lfloor x\rfloor-\frac{1}{2}$. First via (2.4), as $N, \alpha \rightarrow \infty$,

$$
\sum_{k=1}^{N-1} 2 \ln \left(\frac{b}{4}\right)=2 t+\int_{0}^{t} I(8 x) \frac{d x}{x}+O\left(N^{-1}\right)
$$


where the error term is uniform on any finite interval $0 \leq t \leq t_{0}$. Secondly via (2.21)

$$
\begin{aligned}
\sum_{k=1}^{N-1} k(l+2)= & N\left[-t+\frac{2}{t} \int_{0}^{t}(J(8 x)-(1-\ln 2)) d x-\frac{2}{t} \int_{0}^{t} I(8 x) d x+\frac{1}{t} \int_{0}^{t} \ln S(4 x) d x\right] \\
& +t-\frac{3}{2} t^{2}-2 t I(8 t)-\frac{1}{2} I(8 t)-\frac{1}{2} I^{2}(8 t)-\frac{3}{2} I(8 t)-\frac{1}{4} \int_{0}^{t} I^{2}(8 x) \frac{d x}{x} \\
& +2 \int_{0}^{t} J^{\prime}(8 x)\left(4+\frac{2}{x} I(8 x)\right) d x+J(8 t)-(1-\ln 2)+\frac{1}{2} \ln S(4 t)+O\left(N^{-1}\right)
\end{aligned}
$$

and from (2.14),

$$
\sum_{k=1}^{N-1} \frac{v-\frac{7}{6}}{k}=O\left(N^{-1}\right)
$$

as $N, \alpha \rightarrow \infty$, where the error terms are uniform on any finite interval $0 \leq t \leq t_{0}$. We go back to (6.4), as $N, \alpha \rightarrow \infty$,

$$
\sigma_{N, t}=C_{0} N ! e^{N \Phi(t)+\Psi(t)}\left(1+O\left(N^{-1}\right)\right), \quad 0 \leq t \leq t_{0}
$$

with

$$
\Phi(t)=-t+\frac{2}{t} \int_{0}^{t}(J(8 x)-(1-\ln 2)) d x-\frac{2}{t} \int_{0}^{t} I(8 x) d x+\frac{1}{t} \int_{0}^{t} \ln S(4 x) d x
$$

and

$$
\begin{aligned}
\Psi(t)= & 3 t-\frac{3}{2} t^{2}-2 t I(8 t)-\frac{1}{2} I^{2}(8 t)-\frac{3}{2} I(8 t)-\frac{1}{2} \ln S(4 t)-(J(8 t)-(1-\ln 2)) \\
& -\frac{1}{4} \int_{0}^{t} I^{2}(8 x) \frac{d x}{x}+\int_{0}^{t} I(8 x) \frac{d x}{x}+2 \int_{0}^{t}\left(J^{\prime}(8 x)+\frac{S^{\prime}(4 x)}{2 S(4 x)}\right)\left(1+\frac{I(8 x)}{2 x}\right) 4 x d x .
\end{aligned}
$$

The small $t$-behavior of $\Phi(t)$ and $\Psi(t)$ can be determined from (2.4) and (2.19), we have, as $t \rightarrow 0$,

$$
\Phi(t)=\frac{t^{2}}{3}+O\left(t^{3}\right), \quad \Psi(t)=5 t-\frac{9}{2} t^{2}+O\left(t^{3}\right) .
$$

Back to (6.2), we have on one hand

$$
\sigma_{N, t} \sim N ! \quad \text { as } t \rightarrow 0
$$

On the other hand, if we let $N, \alpha \rightarrow \infty$ such that $N t^{2} \rightarrow 0$ (i.e. in particular $t \rightarrow 0$ ), then (6.6) and the behavior of $\Phi(t)$ and $\Psi(t)$ at the origin imply, that

$$
\sigma_{N, t} \sim C_{0} N ! \quad \text { as } N, \alpha \rightarrow \infty: N t^{2} \rightarrow 0 .
$$

Comparing (6.9) with (6.10), this implies

$$
C_{0}=1 \text {, }
$$

and we have therefore shown

Theorem 6.1. In the double scaling limit $N, \alpha \rightarrow \infty$

$$
Z_{N}=N !\left(\frac{\alpha^{2}-1}{2 \alpha}\right)^{N^{2}}\left(\frac{2}{\alpha}\right)^{N} e^{N \Phi(t)+\Psi(t)}\left(1+O\left(N^{-1}\right)\right)
$$


where $\Phi(t)$ and $\Psi(t)$ are given explicitly in (6.7), (6.8) and the error term is uniform on any finite interval $0 \leq t \leq t_{0}$.

The explicit evaluation of the numerical constant $C_{0}$ is crucial for our further strategy. In order to compute the constants $c$ and $d$, we will go back to (6.4), evaluate now the sums in the limit $t \rightarrow \infty$ and then compare the result with (1.15) and (5.3).

\section{Proof of theorem 1.2}

The computations in the last section lead to the following expansion for $\sigma_{N, t}$, as $N \rightarrow \infty$

$$
\sigma_{N, t}=\frac{N !}{1-\tau^{2}} \exp \left[\sum_{k=1}^{N-1}\left(2 \ln \left(\frac{b}{4}\right)+k(l+2)+\frac{v-\frac{7}{6}}{k}\right)\right]\left(1+O\left(N^{-1}\right)\right)
$$

where the error term is uniform with respect to the parameter $0 \leq \tau \leq 1-\varepsilon<1$. In order to derive (1.15) including the constant term, we now evaluate the sums in the last estimation in the limit $N \rightarrow \infty$ as $\alpha>1$ and $t>t_{0}$ (i.e. $t \rightarrow \infty$ ). This time we use the Euler-Maclaurin type summation formula

$$
\sum_{k=1}^{N-1} g(k \tau)=\frac{1}{\tau} \int_{0}^{t} g(x) d x-\frac{1}{2 \tau} \int_{0}^{\tau} g(x) d x-\frac{1}{2 \tau} \int_{t-\tau}^{t} g(x) d x+R
$$

with

$$
R=-\frac{1}{4 \tau} \sum_{k=1}^{N-1} \int_{-\tau}^{\tau} \int_{0}^{x} g^{\prime \prime}(k \tau+u)(x-u) d u d x=O\left(\tau \int_{0}^{t}\left|g^{\prime \prime}(x)\right| d x\right)
$$

which holds for a twice differentiable function $g: \mathbb{R} \rightarrow \mathbb{R}$. To derive formula (7.1), we write the Taylor formula with an integral form for the remainder

$$
g(s+x)=g(s)+g^{\prime}(s) x+\frac{1}{2} \int_{0}^{x} g^{\prime \prime}(s+u)(x-u) d u,
$$

then integrate from $-\tau$ to $\tau$,

$$
\int_{-\tau}^{\tau} g(s+x) d x=2 g(s) \tau+\frac{1}{2} \int_{-\tau}^{\tau} \int_{0}^{x} g^{\prime \prime}(s+u)(x-u) d u d x,
$$

and now sum over $\{s=k \tau, k=1, \ldots, N-1\}$,

$$
\sum_{k=1}^{N-1} \int_{-\tau}^{\tau} g(k \tau+x) d x=2 \sum_{k=1}^{N-1} g(k \tau) \tau+\frac{1}{2} \sum_{k=1}^{N-1} \int_{-\tau}^{\tau} \int_{0}^{x} g^{\prime \prime}(k \tau+u)(x-u) d u d x,
$$

which implies (7.1).

From (2.9) and (2.5), as $N \rightarrow \infty$,

$$
\sum_{k=1}^{N-1} 2 \ln \left(\frac{b}{4}\right)=-2(N-1) \ln (1-\tau)-\ln t+c_{1}+O(\tau)+O\left(t^{-1 / 2}\right)
$$

where we introduced as abbreviation

$$
c_{1}=\int_{0}^{1} I(8 x) \frac{d x}{x}+\int_{1}^{\infty}(I(8 x)+1) \frac{d x}{x}=-3 \ln 2+\int_{0}^{1} I(x) \frac{d x}{x}+\int_{1}^{\infty}(I(x)+1) \frac{d x}{x}=-\ln 2
$$


and the error terms are uniform with respect to the parameters $0 \leq \tau \leq 1-\varepsilon<1$ and $t>t_{0}$. Next, combining (2.9) with (2.14),

$$
v=\frac{7}{6}+O\left(\frac{\tau}{\sqrt{1+t}}\right)
$$

and hence,

$$
\sum_{k=1}^{N-1} \frac{v-\frac{7}{6}}{k}=O(\tau)+O\left(\tau t^{-1 / 2}\right) .
$$

The evaluation of the remaining term involving the Lagrange multiplier will be split into several parts. First

$$
\begin{aligned}
\sum_{k=1}^{N-1} k(l+2)= & N(N-1)+2(1-\ln 2)(N-1)^{2}+\sum_{k=1}^{N-1} \ln S(t b)+\sum_{k=1}^{N-1} 2 J(2 b t) \\
& +\sum_{k=1}^{N-1} k\left(2 \ln b-\frac{b}{2}(1-\tau)-b\right) \\
\equiv & N(N-1)+2(1-\ln 2)(N-1)^{2}+\Sigma_{1}+\Sigma_{2}+\Sigma_{3} .
\end{aligned}
$$

For $\Sigma_{1}$, use the asymptotic formula

$$
\ln S(x)=x-\ln (2 x)+O\left(e^{-2 x}\right), \quad x \rightarrow+\infty
$$

and derive

$$
\begin{aligned}
\Sigma_{1}= & \frac{2 \tau}{1-\tau}(N-1)^{2}-N \ln \left(\frac{8 t}{1-\tau}\right)+N+\sqrt{N} \frac{\zeta(3 / 2)}{\sqrt{2 \pi(\alpha-1)}}+\ln t \\
& +\alpha c_{2}+c_{3}+O(\tau)+O\left(t^{-1 / 2}\right)+O\left(\alpha e^{-8 t}\right),
\end{aligned}
$$

valid in the limit $N \rightarrow \infty, \alpha>1$ with $t \rightarrow \infty$. Here we have

$$
\begin{aligned}
c_{2}= & \int_{0}^{\infty}(\ln S(4 x)-4 x+\ln (8 x)) d x=\frac{1}{8} \int_{0}^{\infty} \ln \left(1-e^{-x}\right) d x=-\frac{\pi^{2}}{48} \\
c_{3}= & \frac{3}{2} \ln 2+\int_{0}^{\infty} 4 x\left(\frac{S^{\prime}(4 x)}{S(4 x)}-1+\frac{1}{4 x}\right)\left(1+\frac{I(8 x)}{2 x}\right) d x+2 \int_{0}^{\infty}\left(I(8 x)+1-\frac{\zeta(3 / 2)}{4 \sqrt{2 \pi x}}\right) d x \\
& -\frac{1}{2} \int_{0}^{1} I(8 x) \frac{d x}{x}-\frac{1}{2} \int_{1}^{\infty}(I(8 x)+1) \frac{d x}{x}=2 \ln 2+\frac{\pi^{2}}{48}+\frac{1}{2} \int_{0}^{\infty} \frac{I(x)}{e^{x}-1} d x,
\end{aligned}
$$

and we simplified the expressions for $c_{i}$, by recalling the definitions of $S(x)$ and $I(x)$ as well as the integrals

$$
\int_{0}^{\infty} \ln \left(1-e^{-x}\right) d x=-\frac{\pi^{2}}{6}, \quad \int_{0}^{\infty} \frac{x d x}{e^{x}-1}=\frac{\pi^{2}}{6} .
$$

Next we go back to Proposition 2.4 and derive

$$
\Sigma_{2}=\alpha c_{4}+c_{5}+O(\tau)+O\left(\alpha t^{-1 / 2}\right)
$$


where

$$
\begin{aligned}
& c_{4}=2 \int_{0}^{\infty} J(8 x) d x=\frac{1}{4} \int_{0}^{\infty} J(x) d x=\frac{\pi^{2}}{48} \\
& c_{5}=-(1-\ln 2)+16 \int_{0}^{\infty} x J^{\prime}(8 x)\left(1+\frac{I(8 x)}{2 x}\right) d x=-(1-\ln 2)-\frac{\pi^{2}}{48}+\int_{0}^{\infty} J^{\prime}(x) I(x) d x,
\end{aligned}
$$

in the limit $N \rightarrow \infty, \alpha>1$, with error terms which are uniform with respect to the parameters $0 \leq \tau \leq 1-\varepsilon<1$ and $t>t_{0}$. Here we have used the definite integrals

$$
\int_{0}^{1}\left(\sqrt{\frac{x}{1-x}}-\arctan \sqrt{\frac{x}{1-x}}\right) \frac{d x}{x^{2}}=\frac{\pi}{2}, \quad \int_{0}^{1}\left(\sqrt{\frac{x}{1-x}}-\sqrt{x}\right) \frac{d x}{x^{2}}=2
$$

in order to simplify the expressions for $c_{i}$. Finally with (2.9) and Proposition 2.1,

$$
\begin{aligned}
\Sigma_{3}= & N(N-1) \ln \left(\frac{4}{1-\tau}\right)-N(N-1)-\frac{2}{1-\tau}(N-1)^{2}-\zeta\left(\frac{3}{2}\right) \alpha \sqrt{\frac{N}{2 \pi(\alpha-1)}} \\
& -\frac{1}{4} \ln t+\alpha c_{6}+c_{7}+O(\tau)+O\left(\alpha t^{-1 / 2}\right)
\end{aligned}
$$

where

$$
\begin{aligned}
c_{6}= & -2 \int_{0}^{\infty}\left(I(8 x)+1-\frac{\zeta(3 / 2)}{4 \sqrt{2 \pi x}}\right) d x=0 \\
c_{7}= & 1-\int_{0}^{1} I^{2}(8 x) \frac{d x}{4 x}-2 \int_{0}^{\infty}\left(I^{\prime}(8 x)+\frac{\zeta(3 / 2)}{4 \sqrt{\pi}(8 x)^{3 / 2}}\right) 8 x\left(1+\frac{I(8 x)}{2 x}\right) d x \\
& +\int_{1}^{\infty}\left(1-I^{2}(8 x)\right) \frac{d x}{4 x}-2 \int_{0}^{\infty}\left(I(8 x)+1-\frac{\zeta(3 / 2)}{4 \sqrt{2 \pi x}}\right) d x \\
& -\frac{\zeta(3 / 2)}{2 \sqrt{2 \pi}} \int_{0}^{\infty}\left(4 I^{\prime}(8 x)-\frac{I(8 x)}{2 x}\right) \frac{d x}{\sqrt{x}}=\frac{1}{2}-\frac{3}{4} \ln 2-\frac{1}{4} \int_{0}^{1} I^{2}(x) \frac{d x}{x}+\frac{1}{4} \int_{1}^{\infty}\left(1-I^{2}(x)\right) \frac{d x}{x} .
\end{aligned}
$$

Now back to (7.4), as $N \rightarrow \infty$,

$$
\begin{aligned}
\sum_{k=1}^{N-1} k(l+2)= & -N^{2} \ln (1-\tau)+2 N \ln (1-\tau)-N \ln (2 t)+N-\sqrt{N} \zeta\left(\frac{3}{2}\right) \sqrt{\frac{\alpha-1}{2 \pi}} \\
& +\frac{3}{4} \ln t+\alpha\left(c_{2}+c_{4}+c_{6}\right)+c_{3}+c_{5}+c_{7}-2 \ln 2 \\
& +O(\tau)+O\left(\alpha t^{-1 / 2}\right)
\end{aligned}
$$

which is uniform with respect to the parameters $0 \leq \tau \leq 1-\varepsilon<1$ and $t>t_{0}$. In order to obtain the desired expansion for $\sigma_{N, t}$ we combine estimations (7.2), (77.3) and (7.5), as $N \rightarrow \infty$

$$
\sigma_{N, t}=\left(\frac{\alpha}{\alpha-1}\right)^{N^{2}}\left(\frac{\alpha}{2}\right)^{N} \hat{C}_{N, t} G^{\sqrt{N}} N^{1 / 4}\left(1+O\left(N^{-1}\right)\right), \quad G=\exp \left[-\zeta\left(\frac{3}{2}\right) \sqrt{\frac{\alpha-1}{2 \pi}}\right]
$$


with

$$
\hat{C}_{N, t}=(\alpha-1)^{1 / 4} \exp \left[d_{0} \alpha+c_{0}+O(\tau)+O\left(\alpha t^{-1 / 2}\right)\right]
$$

where all error terms are uniform with respect to the parameters $0 \leq \tau \leq 1-\varepsilon<1$ and $t>t_{0}$. The factors $d_{0}$ and $c_{0}$ are given as

$$
d_{0}=c_{2}+c_{4}+c_{6}=0
$$

and

$$
\begin{aligned}
c_{0}= & -\frac{1}{2}-\frac{1}{4} \ln 2+\frac{1}{2} \ln \pi \\
& +\frac{1}{2} \int_{0}^{\infty} \frac{I(x)}{e^{x}-1} d x+\int_{0}^{\infty} J^{\prime}(x) I(x) d x-\frac{1}{4} \int_{0}^{1} I^{2}(x) \frac{d x}{x}+\frac{1}{4} \int_{1}^{\infty}\left(1-I^{2}(x)\right) \frac{d x}{x} .
\end{aligned}
$$

We have therefore shown

Proposition 7.1. In the limit $N \rightarrow \infty$,

$$
Z_{N}=\hat{C}_{N, t} F^{N^{2}} G^{\sqrt{N}} N^{1 / 4}\left(1+O\left(N^{-1}\right)\right), \quad F=\frac{\alpha+1}{2}, \quad G=\exp \left[-\zeta\left(\frac{3}{2}\right) \sqrt{\frac{\alpha-1}{2 \pi}}\right]
$$

with

$$
\hat{C}_{N, t}=(\alpha-1)^{1 / 4} \exp \left[c_{0}+O(\tau)+O\left(\alpha t^{-1 / 2}\right)\right],
$$

where $c_{0}$ is given explicitly in (7.7) and the error terms are uniform with respect to the parameters $0 \leq \tau \leq 1-\varepsilon<1$ and $t>t_{0}$.

The last proposition allows us to prove Theorem 1.2. To this end let us choose $\alpha$ from a compact subset of the set

$$
\{\alpha \in \mathbb{R}: \alpha>1\} .
$$

Then, as $N \rightarrow \infty$,

$$
Z_{N}=(\alpha-1)^{1 / 4} e^{c_{0}+O(\tau)} F^{N^{2}} G^{\sqrt{N}} N^{1 / 4}\left(1+O\left(N^{-1 / 2}\right)\right)
$$

where the error term is uniform on any compact subset of the set (1.16). Comparing the last line with (5.3), we obtain

$$
d=0, \quad c=c_{0} .
$$

In order to derive the stated expression for $c$ in Theorem 1.2, we will simplify the integrals appearing in (7.7) as follows. For the last two integrals in (7.7), we replace one of the factors in the products $I^{2}(x)$ with its definition (1.26). Evaluating the integrals and recalling our computations for $c_{1}$, we obtain

$$
\begin{aligned}
-\frac{1}{4} \int_{0}^{1} I^{2}(x) \frac{d x}{x}+\frac{1}{4} \int_{1}^{\infty}\left(1-I^{2}(x)\right) \frac{d x}{x}= & \frac{1}{2} \ln 2-\frac{1}{4 \pi^{2}} \int_{0}^{1} \sqrt{\frac{u}{1-u}} \int_{0}^{1} \sqrt{\frac{v}{1-v}} \\
& \times\left[\int_{0}^{\infty}\left(\frac{1}{e^{x v}-1}-\frac{1}{x v}\right) \frac{x d x}{e^{x u}-1}\right] d u d v .
\end{aligned}
$$

Next, we use geometric progression for $\frac{1}{e^{z}-1}$ and integrate term by term

$$
\int_{0}^{\infty}\left(\frac{1}{e^{x v}-1}-\frac{1}{x v}\right) \frac{x d x}{e^{x u}-1}=\sum_{n=1}^{\infty}\left[-\frac{1}{n u v}+\sum_{m=1}^{\infty} \frac{1}{(n u+m v)^{2}}\right], \quad 0<u, v<1 .
$$


With the help of the integrals

$$
\int_{0}^{1} \sqrt{\frac{u}{1-u}} \frac{d u}{(1+a u)^{2}}=\frac{\pi}{2(1+a)^{3 / 2}}, \quad \int_{0}^{1} \frac{d u}{\sqrt{1-u}(a+u)^{3 / 2}}=\frac{2}{(1+a) \sqrt{a}}, \quad a \geq 0
$$

this implies

$$
-\frac{1}{4} \int_{0}^{1} I^{2}(x) \frac{d x}{x}+\frac{1}{4} \int_{1}^{\infty}\left(1-I^{2}(x)\right) \frac{d x}{x}=\frac{1}{2} \ln 2-\frac{1}{4 \pi} \sum_{n=1}^{\infty}\left[-\frac{\pi}{n}+\sum_{m=1}^{\infty} \frac{1}{(m+n) \sqrt{m n}}\right] .
$$

For the second integral in (7.7) we use the identities

$$
J^{\prime}(x)=I^{\prime}(x)-\frac{1}{\pi} \int_{0}^{1} \arctan \sqrt{\frac{u}{1-u}} \frac{d}{d x}\left(\frac{x}{e^{x u}-1}\right) d u, \quad \frac{\partial}{\partial x}\left(\frac{x}{e^{x u}-1}\right)=\frac{\partial}{\partial u}\left(\frac{u}{e^{x u}-1}\right)
$$

and integrate by parts

$$
\int_{0}^{\infty} J^{\prime}(x) I(x) d x=\frac{1}{2}-\frac{1}{2} \int_{0}^{\infty} \frac{I(x)}{e^{x}-1} d x+\frac{1}{2 \pi} \int_{0}^{\infty} \int_{0}^{1} \sqrt{\frac{u}{1-u}} \frac{I(x)}{e^{x u}-1} d u d x .
$$

Since

$$
I(x)=\frac{x}{\pi} \int_{0}^{1} \sqrt{\frac{v}{1-v}}\left(\frac{1}{e^{x v}-1}-\frac{1}{x v}\right) d v
$$

we obtain

$$
\frac{1}{2 \pi} \int_{0}^{\infty} \int_{0}^{1} \sqrt{\frac{u}{1-u}} \frac{I(x)}{e^{x u}-1} d u d x=\frac{1}{2 \pi^{2}} \int_{0}^{1} \sqrt{\frac{u}{1-u}} \int_{0}^{1} \sqrt{\frac{v}{1-v}}\left[\int_{0}^{\infty}\left(\frac{1}{e^{x v}-1}-\frac{1}{x v}\right) \frac{x d x}{e^{x u}-1}\right] d u d v,
$$

i.e. the triple integral we just evaluated in the computation of (7.9). We summarize

$$
\int_{0}^{\infty} J^{\prime}(x) I(x) d x=\frac{1}{2}-\frac{1}{2} \int_{0}^{\infty} \frac{I(x)}{e^{x}-1} d x+\frac{1}{2 \pi} \sum_{n=1}^{\infty}\left[-\frac{\pi}{n}+\sum_{m=1}^{\infty} \frac{1}{(m+n) \sqrt{m n}}\right]
$$

and back to (7.7),

$$
c=\frac{1}{4} \ln 2+\frac{1}{2} \ln \pi+\frac{1}{4 \pi} \sum_{n=1}^{\infty}\left[-\frac{\pi}{n}+\sum_{m=1}^{\infty} \frac{1}{(m+n) \sqrt{m n}}\right],
$$

thus proving Theorem 1.2

\section{REFERENCES}

[1] D. Allison and N. Reshetikhin, Numerical study of the 6-vertex model with domain wall boundary conditions, Ann. Inst. Fourier (Grenoble) $5 \mathbf{5}$ (2005), 1847-1869.

[2] J. Baik, R. Buckingham and J. DiFranco, Asymptotics of Tracy-Widom distributions and the total integral of a Painlevé II function, Commun. Math. Phys. 280 (2008), 463-497

[3] E. Basor and C. Tracy, Some problems associated with the asymptotics of $\tau$-functions, Surikagaku (Mathematical Sciences) 30, no. 3 (1992), 71-76.

[4] H. Bateman, A. Erdelyi, Higher transcendental functions, McGraw-Hill, NY, 1953.

[5] P. Bleher and T. Bothner, Exact solution of the six-vertex model with domain wall-boundary conditions. Critical line between disordered and antiferroelectric phases, Random Matrices: Theory Appl. 01, (2012) 1250012. 
[6] P. Bleher and V. Fokin, Exact solution of the six-vertex model with domain wall-boundary conditions. Disordered phase, Commun. Math. Phys. 268 (2006), 223-284.

[7] P. Bleher and K. Liechty, Exact solution of the six-vertex model with domain wall-boundary conditions. Ferroelectric phase, Commun. Math. Phys. 286 (2009), 777-801.

[8] P. Bleher and K. Liechty, Exact solution of the six-vertex model with domain wall-boundary conditions. Critical line between ferroelectric and disordered phases, J. Statist. Phys. 134 (2009), 463-485.

[9] P. Bleher and K. Liechty, Exact solution of the six-vertex model with domain wall-boundary conditions. Antiferroelectric phase, Commun. Pure Appl. Math. 63 (2010), 779-829.

[10] P. Bleher and A. Kuijlaars, Large $n$ limit of Gaussian random matrices with external source, part III: Double scaling limit, Commun. Math. Phys. 270 (2007), 481-517.

[11] T. Bothner and A. Its, Asymptotics of a Fredholm determinant corresponding to the first bulk critical universality class in random matrix models, Commun. Math. Phys. 328 (2014), 155-202.

[12] A. Budylin, V. Buslaev, Quasiclassical asymptotics of the resolvent of an integral convolution operator with a sine kernel on a finite interval, (Russian) Algebra i Analiz 7, no. 6 (1995), 79-103

[13] D. A. Coker, A. G. Izergin and V. E. Korepin, Determinant formula for the six-vertex model, J. Phys. A 25, (1992) 4315-4334.

[14] P. Deift, A. Its, I. Krasovsky and X. Zhou, The Widom-Dyson constant for the gap probability in random matrix theory, J. Comput. Appl. Math. 202 no. 1 (2007), 26-47

[15] P. Deift, A. Its and I. Krasovsky, Asymptotics of the Airy-kernel determinant, Commun. Math. Phys. 278 no. 3 (2008), 643-678

[16] P. A. Deift, T. Kriecherbauer and K. T-R. McLaughlin, New results on equlibirum measure for logarithmic potentials in the presence of an external field, J. Approx. Theory 95 (1998), 388-475.

[17] P. A. Deift, T. Kriecherbauer, K. T-R. McLaughlin, S. Venakides and X. Zhou, Uniform asymptotics for polynomials orthogonal with respect to varying exponential weights and applications to universality questions in random matrix theory, Commun. Pure Appl. Math. 52 (1999), 1335-1425.

[18] P. A. Deift and X. Zhou, A steepest descent method for oscillatory Riemann-Hilbert problems. Asymptotics for the MKdV equation, Ann. of Math., 137 (1993), 295-368.

[19] T. Ehrhardt, Dyson's constant in the asymptotics of the Fredholm determinant of the sine kernel, Commun. Math. Phys. 262 (2006), 317-341

[20] P. L. Ferrari and H. Spohn, Domino tilings and the six-vertex model at its free fermion point, J. Phys. A: Math. Gen. 39 (2006) 1029710306.

[21] A. S. Fokas, A. R. Its and A. V. Kitaev, Discrete Painlevé equations and their appearance in quantum gravity, Comm. Math. Phys. 142 (2) (1991), 313-344.

[22] P. Forrester, Asymptotics of spacing distributions 50 years later, to appear in Proceedings of the MSRI semester "Random matrix theory, interacting particle systems and integrable systems", preprint: arXiv: 1204.3225v3

[23] A. G. Izergin, Partition function of the six-vertex model in a finite volume. Dokl. Akad. Nauk SSSR 297, no. 2, (1987), 331-333; translation in Soviet Phys. Dokl. 32 (1987), 878-880.

[24] V. Korepin, Calculation of norms of Bethe wave functions, Commun. Math. Phys. 86 (1982), 391-418.

[25] V. Korepin and P. Zinn-Justin, Thermodynamic limit of the six-vertex model with domain wall boundary conditions, J. Phys. A 33(40), (2000) 7053.

[26] C. Tracy, Asymptotics of the $\tau$-function in the two-dimensional Ising model, Commun. Math. Phys. 142 (1991), 297-311.

[27] M. Vanlessen, Strong asymptotics of Laguerre-type orthogonal polynomails and applications in random matrix theory, Construct. Approx. 25 (2007), 125-175.

[28] P. Zinn-Justin, Six-vertex model with domain wall boundary conditions and one-matrix model, Phys. Rev. E 62, (2000) 3411-3418.

Department of Mathematical Sciences, Indiana University-Purdue University Indianapolis, 402 N. BlaCKFord St., Indianapolis, IN 46202, U.S.A.

E-mail address: bleher@math.iupui.edu

Department of Mathematical Sciences, Indiana University-Purdue University Indianapolis, 402 N.

BlackFord St., IndianApolis, IN 46202, U.S.A.

E-mail address: tbothner@iupui.edu 